\title{
Micro QuEChERS-based method for the simultaneous biomonitoring in whole blood of 360 toxicologically relevant pollutants for wildlife
}

\author{
Cristian Rial-Berriel ${ }^{\mathrm{a}, \mathrm{b}, 1}$, Andrea Acosta-Dacal ${ }^{\mathrm{a}, 1}$, Manuel Zumbado ${ }^{\mathrm{a}, \mathrm{c}}$, Octavio P. Luzardo ${ }^{\mathrm{a}, \mathrm{b}, \mathrm{c}, *}$ \\ ${ }^{a}$ Toxicology Unit, Research Institute of Biomedical and Health Sciences (IUIBS), Universidad de Las Palmas de Gran Canaria, Paseo Blas Cabrera s/n, 35016 Las Palmas de Gran Canaria, Spain \\ b Study Group on Wild Animal Conservation Medicine (GEMAS), Spain \\ ' Spanish Biomedical Research Center in Physiopathology of Obesity and Nutrition (CIBERObn), Spain
}

\section{H I G H L I G H T S}

- Validation of a method for the simultaneous quantification of 360 toxic chemicals in whole blood

- One-step acetate buffered micro QuEChERS using acidified acetonitrile yielded recoveries $>70 \%$.

- Only $250 \mu \mathrm{l}$ of sample and quantification at the sub-ppb level makes it suitable for biomonitoring.

- Verified in a series of 36 barn owls and 112 common kestrels, which test positive for 3-25 pollutants

\section{A R T I C L E I N F O}

\section{Article history:}

Received 25 March 2020

Received in revised form 12 May 2020

Accepted 12 May 2020

Available online 15 May 2020

Editor: Yolanda Picó

\section{Keywords:}

Persistent organic pollutants

Pharmaceuticals

Pesticides

Rodenticides

Raptors

Mass spectrometry

\section{G R A P H I C A L A B S T R A C T}

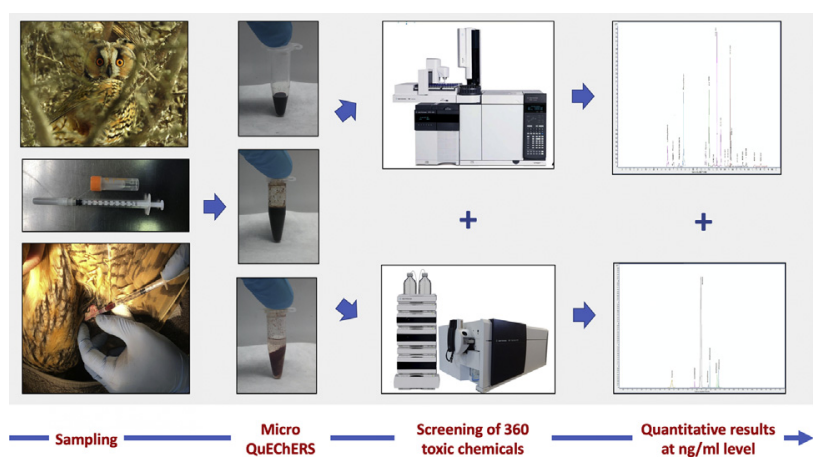

\begin{abstract}
A B S T R A C T
This work presents the optimization, validation, and verification of a miniaturized method for the determination of 360 environmental pollutants that are of toxicological concern for wildlife. The method implies a one-step QuEChERS-based extraction of $250 \mu \mathrm{l}$ whole blood using acidified acetonitrile, followed by two complementary analyses by LC-MS/MS and GC-MS/MS. The optimized conditions allow the simultaneous determination of the major persistent organic pollutants, a wide range of plant protection products, rodenticides, pharmaceuticals, and a suite of metabolites that can be used as biomarkers of exposure. The method is very sensitive, and $95 \%$ of the pollutants can be detected at concentrations below $1.5 \mathrm{ng} / \mathrm{ml}$. The method was applied to a series of 148 samples of nocturnal and diurnal wild raptors collected during field ecological studies in 2018 and 2019. Fiftyone different contaminants were found in these samples, with a median value of 7 contaminants per sample. As expected, five of the six contaminants that were detected in $>50 \%$ of the samples were persistent or semipersistent organic pollutants. However, it is striking the high frequency of detection of some non-persistent pollutants, such as 2-phenylphenol, benalaxyl, metaflumizone, diphenylamine, brodifacoum or levamisole, indicating the penetration of these chemicals into the food chains. The toxicological significance of all these findings should be studied in depth in future research. However, the results clearly demonstrated that the approach developed provides reliable, simple, and rapid determination of a wide range of pollutants in wildlife and makes it very useful to obtain valuable data in biomonitoring studies with only small amounts of sample.

(C) 2020 Elsevier B.V. All rights reserved.
\end{abstract}

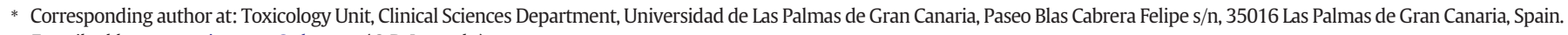
E-mail address: octavio.perez@ulpgc.es (O.P. Luzardo).

1 These authors contributed equally to the work, and therefore should be considered indistinctly as first authors.
} 


\section{Introduction}

In environmental chemistry, biomonitoring is the procedure by which the body burden of toxic or potentially toxic chemicals, in living beings, is assessed as a means of exposure assessment. Blood, its fractions, and urine samples are the most common samples, but tissues and other fluids can also be used, such as hair, feathers, or breast milk, among others (Haines et al., 2017; Ibarluzea et al., 2016).

A recent report, has indicated that since 1974 there has been a 60\% decrease in wildlife populations worldwide (WWF, 2018). This alarming decrease responds to multiple causes, among which are the change in land uses, the destruction of the habitat of many species, the climate change, but also the penetration of chemistry in ecosystems (Hernout et al., 2011). A clear and very well documented example of the latter, is found in the drastic decline in populations of Asian vultures due to exposure to diclofenac, widely used as a veterinary antiinflammatory drug, and that in these birds, produces lethal nephropathy (Sathishkumar et al., 2020). The amount and variety of chemical risks that wildlife faces are enormous, and for many of these pollutants, we have limited knowledge of the potential pressures on wildlife (Hernout et al., 2011).

One of the best-studied chemical groups in wildlife is that of the persistent organic pollutants (POPs, mainly organohalogenated compounds), as these compounds have been linked for decades to population declines, diseases or abnormalities in several species, including certain types of fish, birds, and mammals (Luzardo et al., 2014b; Malarvannan et al., 2020). Besides, their monitoring in wildlife seems to be important because certain animal species can also act as sentinels for human health (Bucchia et al., 2015; Elliott et al., 2018; Fox, 2001; Henriquez-Hernandez et al., 2017; Luzardo et al., 2014a; Reif, 2011), and also for identifying trends in levels that can assess the effectiveness of international control measures (Malarvannan et al., 2020).

In addition to all these legacy pollutants, many other chemicals are of concern for wildlife conservation because of their current or recent extensive use, their high toxicity, their stability, and their relatively high half-life. It is the case of some pesticides, pharmaceuticals, or anticoagulant rodenticides, among others. As regards pesticides, most of them are pollutants from the agricultural sector, where dozens of compounds of multiple chemical classes are used massively (Liao et al., 2019). If we take as a reference the list of substances included in the monitoring programs for residues in food for human consumption, at least 200 chemicals should be considered as worrisome in the European Union (EC, 2019a). Unfortunately, the excessive use of these agricultural pesticides causes their penetration into ecosystems and can compromise the health and even survival of many biological species (Encarnacao et al., 2019; Klich et al., 2020; Krief et al., 2017; Plaza et al., 2019). Also, deliberate abuse of pesticides to poison wildlife occurs throughout the world (Bille et al., 2016; Fajardo et al., 2012; Hernandez and Margalida, 2008; Motas-Guzman et al., 2003; Ntemiri et al., 2018; Ogada, 2014; Ruiz-Suarez et al., 2015). Therefore, in wildlife samples, it is also interesting to monitor not only legal but also frequently employed banned compounds (Luzardo et al., 2014c). A special case of pesticides is that of rodenticides, which are employed extensively around the world, leading to unintended exposure of non-target animals, especially raptors (Nakayama et al., 2019; Ruiz-Suarez et al., 2014).

As well as pesticides, pharmaceuticals have the potential to bioaccumulate and transfer through trophic webs and may threaten wildlife health. Wildlife exposure to pharmaceuticals can occur through contaminated water (Obimakinde et al., 2017), agricultural soils, plants and arthropods (Arnold et al., 2014; Bartikova et al., 2015), and through the excreta and carcasses of medicated livestock (i.e., supplementary feeding of threatened avian scavengers) (Blanco et al., 2017; Cuthbert et al., 2014).

The challenge of detecting such a variety of potentially harmful substances in samples from wild animals, is compounded by the fact that the amount of sample available is often small. Blood can be used as a non-lethal simple sampling matrix, but the sample volume is limited by body size, especially in the case of tiny animals such as songbirds or lizards. Therefore, it is desirable to have robust and sensitive analytical methods, and that these are as miniaturized as possible to maximize the information that can be obtained from a field sampling on a wildlife species. Many authors have published methods for the analysis of pesticides in wildlife samples, and some of them are based on the QuEChERS method. However, most of them have been designed for the analysis of a relatively low number of compounds belonging to the same chemical group (Allender and Keegan, 1992; Brown et al., 1996; Brown et al., 2005; Bucchia et al., 2015; Sage et al., 2010; Taliansky-Chamudis et al., 2017). This makes it necessary to use several of these methods in a complementary manner to biomonitoring all the relevant environmental chemicals, which is often impossible due to sample limitation. For this reason, sensitive and specific multi-residue techniques covering a wide spectrum of toxic or potentially toxic environmental pollutants, can substantially contribute to minimizing the costs and maximizing the chance of assessing the exposure of wildlife to most of the relevant chemicals. Thus, some other authors have developed multi-residue and multi-class methods for the determination of drugs (Qie et al., 2019), or of pesticides (Shin et al., 2018; Srivastava et al., 2017), or of POPs in blood (Vijayasarathy et al., 2019), even some using a very small amount of sample (Shin et al., 2018). However, to our knowledge, none has been developed for the simultaneous detection of contaminants for all of these groups together.

We have developed a multi-class multi-residue method comprising a single-step QuEChERS-based extraction of whole blood and two complementary chromatographic analyses coupled to mass spectrometry. The method allows the simultaneous quantification of 360 toxic or potentially toxic chemicals (POPs, agricultural pesticides, pharmaceuticals, and AR) at the sub-part-per-billion level using only $250 \mu$ of whole blood. Additionally, we present data on environmental exposure to pollutants of 148 chicks belonging to two species of birds of prey from the central region of the Iberian Peninsula (common kestrels and barn owls) and discuss their ecological implications.

\section{Materials and methods}

\subsection{Chemicals, reagents, and biological material}

Certified standards of all the individual pollutants and deuterated compounds (P-ISs, procedural internal standards) which were initially tested (purity 93.1 to 99.8\%), were obtained from Dr. Ehrestorfer (Augsburg, Germany), CPA Chem (Stara Zagora, Bulgaria), A2S - Analytical Standard Solutions (Staint Jean D'Illac, France), Sigma-Aldrich (Augsburg, Germany), Accustandard (New Haven, USA), and European Pharmacopoeia Reference Standards (Strasbourg, France). Salts for extraction based on the QuEChERS method (Quick, Easy, Cheap, Effective, Rugged, and Safe) were purchased in commercial premixes from Agilent Technologies (Palo Alto, USA) in two formats: QuEChERS Extract Pouch, AOAC Method ( $6 \mathrm{~g}$ de magnesium sulfate and $1.5 \mathrm{~g}$ sodium acetate) and QuEChERS Extract Pouch, EN Method (4 g magnesium sulfate, $1 \mathrm{~g}$ sodium chloride, $1 \mathrm{~g}$ sodium citrate dihydrate, and $0,5 \mathrm{~g}$ sodium hydrogencitrate sesquihydrate). QuEChERs dSPE Enhanced Matrix Removal-Lipid (EMR-lipid; Agilent, Palo Alto, USA) was used as a clean-up step in the optimization process. Acetonitrile (ACN, 99.9\% purity), methanol (MeOH, 99.9\% purity), and formic acid (FA, 98.0\% purity) were purchased from Honeywell (Charlotte, USA), and were of LC-MS grade. The water for preparing the mobile phase $(18.2 \mathrm{M} \Omega / \mathrm{cm})$ was obtained using an Elix Advantage 15UV tandem coupled to a MilliQ A10 Gradient system (Millipore, Molsheim, France). Ammonium acetate and ammonium formate were purchased from Fisher (Fisher Scientific UK, Loughborough, UK), and was of Optima LC-MS grade.

For the development, optimization, and validation of the analytical technique, we employed blood samples obtained from chickens and 
goats from the animal housing facilities of the Faculty of Veterinary of the University of Las Palmas de Gran Canaria. All the animals were born in this facility, were healthy and had never been exposed to chemicals (no farms or agricultural facilities in the nearby, and no pharmacological treatments in the last two months), to avoid drug interference. Whole blood was obtained by puncturing the brachial vein (chickens, 23G needle) or the jugular vein (goats, 20G needle), using 3.5 ml-vacutainer tubes with heparin as anticoagulant. Upon arrival at the laboratory, aliquots were homogenized, pooled (by species), and stored at $-24{ }^{\circ} \mathrm{C}$ until use.

To verify the applicability of the validated method to real samples, we studied a series of 148 blood samples. The samples were obtained from a diurnal and a nocturnal species of raptors (Falco tinnunculus and Tyto alba) and were collected during an ecological field study on the impact on wildlife of the treatment with rodenticides against a common vole (Microtus arvalis) plague. The samples were obtained from nest boxes located in the provinces of Palencia, Salamanca, Burgos, Segovia, Valladolid and Zamora (Castilla-León, Spain). All samples were collected after obtaining the corresponding permits and following the animal welfare protocols during the sampling (Espin et al., 2016).

\subsection{Stock solutions, calibration standards and quality controls}

Stock solutions of all POPs, pesticides, AR, pharmaceuticals, metabolites, and P-ISs were prepared by dissolving an accurately weighed amount in the suitable solvent ( $\mathrm{ACN}, \mathrm{MeOH}$, water, acetone) to obtain a concentration of either 1 or $0.5 \mathrm{mg} / \mathrm{ml}$. These stock solutions were stored in aliquots at $-32{ }^{\circ} \mathrm{C}$ until use (maximum 1 year). Three intermediate working solutions were prepared by combining the individual standards (by groups: pesticides, pharmaceuticals and POPs, to avoid interferences between compounds and solvents), to give a concentration of $1 \mu \mathrm{g} / \mathrm{ml} /$ each and stored at $-32{ }^{\circ} \mathrm{C}$. Those solutions were renewed every three months. Deuterated standards were prepared separately in the same way in one mixture for both, GC and LC. Calibration standards were made from independent intermediate solutions of the stock solution and spiked in the chicken-, goat-, or combined blood to obtain 12 calibration standards in the range of 0.1 to $20 \mathrm{ng} / \mathrm{ml}$. These matrix-matched calibration standards were freshly prepared for each experiment (daily). Quality controls (QC) samples were made in the same way to obtain three different levels $(0.2,2$, and $10 \mathrm{ng} / \mathrm{ml})$ of all the chemicals. Blank matrix samples were prepared to calculate linearity, matrix effect, carryover, interferences, and stability.

\subsection{Instrumental analysis}

For the detection and quantification of the 360 analytes finally included in this procedure, it is necessary to perform two complementary chromatographic analyses from the blood extract: a liquid chromatography analysis coupled to triple quadrupole mass spectrometry (LCMS/MS) and an analysis by gas chromatography coupled to triple quadrupole mass spectrometry (GC-MS/MS).

\subsection{1. $L C-M S / M S$}

An Agilent 1290 UHPLC tandem coupled to an Agilent 6460 mass spectrometer (Agilent Technologies, Palo Alto, USA) was employed for the analysis of 234 chemicals. The chromatographic separations were performed using an InfinityLab Poroshell $120(2.1 \mathrm{~mm} \times 100 \mathrm{~mm}$, $2.7 \mu \mathrm{m})$. Agilent 1290 Infinity II Inline Filter with $0.3 \mu \mathrm{m}$ SS frit, and Agilent InfinityLab Poroshell 120 UHPLC Guard column $(2.1 \mathrm{~mm} \times 5 \mathrm{~mm}$, $2.7 \mu \mathrm{m}$ ) were used to protect the column. The mobile phase A consisted on $2 \mathrm{mM}$ ammonium acetate and $0.1 \%$ FA in ultrapure water, while the mobile phase B consisted on $2 \mathrm{mM}$ ammonium acetate in $\mathrm{MeOH}$. The mobile phase A gradient was: 95\% - $0.5 \mathrm{~min}$; $80 \%$ - $1 \mathrm{~min}$; $60 \%$ $2.5 \mathrm{~min} ; 15 \%$ - $8 \mathrm{~min}$; $0 \%$ - 10 to $14 \mathrm{~min}$; $95 \%$ - $14.01 \mathrm{~min}$. The flow rate was set at $0.4 \mathrm{ml} / \mathrm{min}$. The injection volume was $8 \mu \mathrm{l}$. The column oven temperature was set at $50{ }^{\circ} \mathrm{C}$. Total run time was $18 \mathrm{~min}$. The mass spectrometer was operated in the dynamic multiple reaction monitoring (dMRM) mode. The optimized operating conditions of the mass spectrometer analyses, in positive and negative, electrospray ionization (Agilent Jet Stream Electrospray Ionization Source, AJS-ESI) were the following: gas temperature $190{ }^{\circ} \mathrm{C}$; nebulizer gas flow $11 \mathrm{l} / \mathrm{min}$; nebulizer pressure $26 \mathrm{psi}$; sheath gas temperature $330{ }^{\circ} \mathrm{C}$; sheath gas flow $12 \mathrm{l} / \mathrm{min}$; capillary voltages $3900 \mathrm{~V}$ (positive), $2600 \mathrm{~V}$ (negative); cycle time $800 \mathrm{~ms}$; dwell time 8-60 ms. Nitrogen provided by Zefiro 40 nitrogen generator (F-DGSi, Evry, France) was used as drying and desolvation gas. Nitrogen 6.0 (99.9999\% purity, Linde, Dublin, Ireland) was used as collision gas.

\subsubsection{GC-MS/MS}

An Agilent 7890B gas chromatographer equipped with an Agilent 7693 automatic sampler and tandem coupled to an Agilent 7010 mass spectrometer (Agilent Technologies, Palo Alto, USA) was employed for the analysis of 126 chemicals. A $1.5 \mu \mathrm{l}$ aliquot of the sample extract was injected on an ultra-inert glass wool inlet liner in pulsed splitless mode. Inlet temperature was set at $250{ }^{\circ} \mathrm{C}$. The chromatographic separations were performed using two fused silica ultra-inert capillary columns Agilent J\&W HP-5MS (crosslinked 5\% phenyl-methylpolysiloxane, Agilent Technologies), each with a length of $15 \mathrm{~m}$, $0.25 \mathrm{~mm}$ i.d., and a film thickness of $0.25 \mu \mathrm{m}$. The use of two $15-\mathrm{m}$ columns allowed the use of the backflushing technique. Both columns were connected by a Purged Ultimate Union (PUU; Agilent Technologies). Helium (99.999\%) was set in constant flow mode as carrier gas, and the flow was adjusted by the retention time lock feature using chlorpyrifos methyl as a reference (reference time $=9.143 \mathrm{~min}$ ). Nitrogen 6.0 (99,9999\% purity, Linde, Dublin, Ireland) was used as collision gas. The oven temperature program was programmed as follows: (a) $80{ }^{\circ} \mathrm{C}$ held for $1.8 \mathrm{~min}$; (b) increase to $170^{\circ} \mathrm{C}$ at a rate of $40{ }^{\circ} \mathrm{C} /$ min; (c) increase to $310{ }^{\circ} \mathrm{C}$ at a rate of $10{ }^{\circ} \mathrm{C} / \mathrm{min}$ to $310{ }^{\circ} \mathrm{C}$; (d) $3 \mathrm{~min}$ hold time at $310{ }^{\circ} \mathrm{C}$. The final run time was $21.05 \mathrm{~min}$. Post-run backflush was set at $-5.8 \mathrm{ml} / \mathrm{min}, 315^{\circ} \mathrm{C}$ for $5 \mathrm{~min}$. The mass spectrometer was operated in the multiple reaction monitoring (MRM) mode, using 24-time segments. The optimized operating conditions of the mass spectrometer analyses were the following: electron impact (EI) ionization source temperature $280^{\circ} \mathrm{C}$; collision gas flow $1.5 \mathrm{ml} / \mathrm{min}$; transfer line temperature $280{ }^{\circ} \mathrm{C}$; solvent delay $3.7 \mathrm{~min}$; cycle time between 300 and 600 ms; dwell time between 15 and 40 ms.

\subsection{Sample preparation}

The optimized extraction protocol consisted of the modification and miniaturization of the QuEChERS method (Anastassiades et al., 2003). For the simultaneous extraction of 360 chemicals, the final extraction protocol was as follows: whole blood samples $(250 \mu \mathrm{l})$ were placed into a $2 \mathrm{ml}$ Eppendorf tube. At this point, the fortification of blank matrix samples for a matrix-matched 12-point calibration curve, was done using different volumes of intermediate fortification solutions for each calibration point. Ten microliters of the mixture of P-ISs, which included compounds used for both, GC (acenaphthene-d10, chlorpyrifos-d10, chrysene-d12, diazinon-d10, PCB 200, and phenanthrene-d10) and LC (atrazine-d5, carbendazim-d3, cyromazine-d4, diazinon-d10, linuron$\mathrm{d} 3$, and pirimicarb-d6) were added to all samples and calibration points to yield a final concentration of $1 \mathrm{ng} / \mathrm{ml}$. The samples were vortexmixed for $30 \mathrm{~s}$, and placed in an orbital shaker for $1 \mathrm{~h}$, to ensure the adequate dispersion and homogenization of the analytes with the blood components. After that, $500 \mu \mathrm{l}$ of acidified acetonitrile (1\% FA) were added, and the tubes were well vortexed for $30 \mathrm{~s}$. Then, the tubes were placed in an ultrasonic bath (Selecta, Barcelona, Spain) at room temperature for $20 \mathrm{~min}$. After that, anhydrous magnesium sulfate $(150 \mathrm{mg})$ and sodium acetate $(37.5 \mathrm{mg}$ ) were added to each tube and thoroughly mixed using vortex for $30 \mathrm{~s}$, and then, vigorous-manually shaken for $1 \mathrm{~min}$. Finally, the samples were microcentrifuged (4200 rpm, $5 \mathrm{~min}$ ) using an ALC 4214 microcentrifuge (A.L.C. 
International SRL, Cologno Monzese, Italy). The supernatant (approximately $400 \mu \mathrm{l}$ ) was collected with a $1-\mathrm{ml}$ syringe, passed through a $0.2 \mu \mathrm{m}$ Chromafil PET-20/15 MS syringe filter (polyester, HPLC certified, Macherey-Nagel, Düren, Germany), and placed in an amber inserted chromatographic vial. This vial was used directly in two consecutive analyses by GC-MS/MS and LC-MS/MS, without the need for further clean-up, dilution or solvent change steps.

\subsection{Assay validation procedures}

The main objective of the validation was to demonstrate the reliability and performance of the method, developed and applied to the whole blood matrix. Initially, chicken and goat blood were tested separately. Still, since there were no significant differences in the performance of the extraction procedures, the complete validation was done with a mixture of the two types of blood, as recommended (EC, 2019b; SWGTOX, 2013). The method validation was performed using the following parameters: identity, selectivity, linearity (as a working range), accuracy (as bias and precision), carryover, interferences and LOQ. The assessment of the matrix effect was also carried out. In general, we followed the recommendations contained in the SANTE guide (EC, 2019b). Since this guide is mainly aimed at the analysis of pesticides in food and feed samples, we have also taken into account the recommendations contained in the guide of Standard Practices for Method Validation in Forensic Toxicology (SWGTOX, 2013), mainly with regard to the particularities of working with the whole blood matrix and pharmaceuticals. All validation assays involve adding known concentrations of analytes to the matrix. However, given the enormous amount of substances included in the method, the whole blood was not completely free of $100 \%$ of the chemicals, in particular the POPs. Therefore, the response of the white matrix sample was subtracted from calibration standards and QC to calculate the response of the analyte added externally. All the details of the final method validation are generally described in the Results and Discussion section. Still, the data for each compound are summarized in Table 1 of the accompanying Data in Brief article entitled "Supporting dataset on the method validation of micro QuEChERS-based method for the simultaneous biomonitoring in whole blood of 360 toxicologically relevant pollutants for wildlife and results in 148 real samples (Falco tinnunculus and Tyto alba)".

\subsection{Statistical analysis}

Both, within-run and between-run precisions, were calculated using the one-way ANOVA approach with the run number (usually $n=5$ ) as the grouping variable. The ANOVA calculations were done using the GraphPad Prism v6.0 (GraphPad Software, CA, USA).

\section{Results and discussion}

\subsection{Optimization of MS/MS conditions and chromatography}

The mass spectrometry conditions were optimized for the detection and quantification of 360 compounds ( 234 by LC-MS/MS and 126 by GC-MS/MS).

\subsection{1. $L C-M S / M S$}

For the mass spectrometry optimization of each compound analyzed by LC-MS/MS, individual chromatographic vials with a concentration of around $100-200 \mathrm{ng} / \mathrm{ml}$ were prepared. The mobile phase conditions were established based on the literature and methodologies previously developed in our laboratory (Luzardo et al., 2015; Luzardo et al., 2013; Luzardo et al., 2014c; Ruiz-Suarez et al., 2015; Ruiz-Suarez et al., 2014). A stainless steel zero dead volume union was used to replace the chromatographic column. The optimization of the precursor ion signal and the product ion signal was conducted manually, as well as the optimization of the fragmentation and collision energy. The best combination of two MRM transitions was selected for each compound (Table 1). Once the list of transitions for all target compounds and PISs was completed, the optimization steps for gas temperature, gas flow, nebulizer gas pressure, sheath gas flow and temperature, capillary voltage $(+/-)$, and nozzle voltage for the AJS-ESI, were performed sequentially using the Mass Hunter Source Optimizer software (Agilent Technologies, Palo Alto, USA).

During the early stage of the chromatographic method development, the suitability and performance of the two different columns were assessed. Both columns are reversed-phased but with slightly different specifications. The first column tested was the ZORBAX Eclipse Plus C18 $(2.1 \mathrm{~mm} \times 50 \mathrm{~mm}, 1.8 \mu \mathrm{m})$, that produced a very broad peak with severe peak tailing for many target analytes. The second column, which we routinely use, the Agilent InfinityLab Poroshell 120 ( $2.1 \mathrm{~mm} \times 100 \mathrm{~mm}, 2.7 \mu \mathrm{m})$, showed an exceptional narrow peak for nearly all target analytes based on the shape of a Gaussian peak. In addition, we tested two different chromatography conditions (mobile phase and gradient) based on our previous experience on the analysis of pesticides and ARs by LC-MS/MS. Condition A: 0.1\% FA and $2 \mathrm{mM}$ ammonium formate in both, water and methanol; condition B: $2 \mathrm{mM}$ ammonium acetate in both, water and methanol. We observed that FA was not optimum for ARs, which performed better in the absence of acid. However, FA proved to be necessary for the analysis of many pesticides, so a compromise was adopted, and we continue to use the acid. The different experiments showed that a sufficient degree of ionization for the analysis of all the compounds (positive/negative), was obtained at $2 \mathrm{mM}$ ammonium acetate (water and methanol), with an optimal percentage of $0.1 \%$ formic acid in water, nor in methanol. In the last step, a series of experiments were conducted to select the optimal volume of injection, which was finally set in $8 \mu$.

\subsubsection{GC-MS/MS}

Acquisition method for GC-MS/MS compounds was initially supplied by Agilent, but it was further optimized in our laboratory. A sequence of injections was programmed to determine the optimal collision energies in increments of $5 \mathrm{eV}$ (range from 5 to $60 \mathrm{eV}$ ); the results of this optimization are also reflected in Table 1, where the analytical parameters of the complete list of chemicals (in alphabetical order) has been summarized. Dwell time and cycle time were also optimized. In GC, no optimization was made in relation to the column type or the temperature program, since our group had previous experience on the separation of many of these compounds or very similar combinations (Bucchia et al., 2015; Luzardo et al., 2015; Luzardo et al., 2014c).

However, since ACN extracts are injected in this method, we did carry out a series of experiments to optimize the solvent delay time, the initial temperature of the oven $\left(60\right.$ to 90 , in increments of $10^{\circ} \mathrm{C}$ ), the temperature of the injector (range of 230 to $300{ }^{\circ} \mathrm{C}$, in increments of $10^{\circ} \mathrm{C}$ ), the temperature of the ionization source (range of 250 to $320^{\circ} \mathrm{C}$, in increments of $10^{\circ} \mathrm{C}$ ), the temperature of the transfer line (from 270 to $320^{\circ} \mathrm{C}$, in increments of $10^{\circ} \mathrm{C}$ ), and the injection volume (from 0.8 to $1.8 \mu \mathrm{l}$, in increments of $0.2 \mu \mathrm{l}$ ). These experiments were performed by injecting a mixture of all the analytes in acetonitrile at two concentrations ( $1 \mathrm{ng} / \mathrm{ml}$ and $50 \mathrm{ng} / \mathrm{ml}$ ), and the parameters that gave the best shape and peak intensity for most of the compounds were selected.

\subsection{Optimization of sample preparation}

The previous experience of our laboratory in the development and application of multi-residue methods made us opt for the QuEChERS method. Two widely available modifications of this method were compared ( 3 replicates at two concentrations $(2-20 \mathrm{ng} / \mathrm{ml})$, analyzed in duplicate): the AOAC Official Method 2007.01 (Lehotay et al., 2010); and the UNE-EN 15662:2019 Official Method (EN, 2019). Both methodologies employ acetonitrile as the extraction solvent. We decided not to test others as acetonitrile has proved to be the solvent with the highest 
Table 1

List of compounds analyzed in whole blood together with the category of use, legal status, the technique employed, and the instrumental conditions of the optimized methods.

\begin{tabular}{|c|c|c|c|c|c|c|c|c|c|c|c|c|c|}
\hline \multirow[t]{2}{*}{ No. } & \multirow[t]{2}{*}{ Compound } & \multirow[t]{2}{*}{ Category $^{\mathrm{a}}$} & \multirow{2}{*}{$\begin{array}{l}\text { Legal } \\
\text { status } \\
\text { in the } \mathrm{EU}^{\mathrm{b}}\end{array}$} & \multirow{2}{*}{$\begin{array}{l}\text { Subjected } \\
\text { to } \mathrm{MRL}^{\mathrm{c}}\end{array}$} & \multirow[t]{2}{*}{ Technique $^{\mathrm{d}}$} & \multirow[t]{2}{*}{ Retention time (min) } & \multirow{2}{*}{$\begin{array}{l}\text { LOQ } \\
(\mathrm{ng} / \mathrm{ml})\end{array}$} & \multirow[t]{2}{*}{ Polarity } & \multicolumn{2}{|l|}{ Quantification } & \multicolumn{2}{|l|}{ Confirmation } & \multirow{2}{*}{$\begin{array}{l}\text { Fragmentor } \\
\text { voltage (V) }\end{array}$} \\
\hline & & & & & & & & & $\begin{array}{l}\text { MRM transition } \\
(\mathrm{m} / \mathrm{z})\end{array}$ & $\begin{array}{l}\text { Collision } \\
\text { energy } \\
(\mathrm{eV})\end{array}$ & $\begin{array}{l}\text { MRM } \\
\text { transition } \\
(m / z)\end{array}$ & $\begin{array}{l}\text { Collision } \\
\text { energy } \\
(\mathrm{eV})\end{array}$ & \\
\hline 1 & 2-Phenylphenol & $\mathrm{F}$ & Approved & Yes & GC & 6.28 & 0.20 & Positive & $169.0 \rightarrow 115.0$ & 30 & $169.0 \rightarrow 141.0$ & 15 & 70 \\
\hline 2 & 4,4'-Dichlorobenzophenone (metabolite of dicofol) & Met & - & No & GC & 9.99 & 0.80 & Positive & $250.0 \rightarrow 139.0$ & 15 & $250.0 \rightarrow 215.0$ & 5 & 70 \\
\hline 3 & Abamectine & $\mathrm{I}, \mathrm{A}, \mathrm{AH}$ & Approved & Yes & LC & 10.99 & 4.00 & Positive & $890.5 \rightarrow 567.1$ & 10 & $895.5 \rightarrow 751.4$ & 45 & 160 \\
\hline 4 & Acenaphthene & POP & - & No & GC & 6.15 & 0.20 & Positive & $153.0 \rightarrow 152.0$ & 25 & $153.0 \rightarrow 151.0$ & 35 & 70 \\
\hline 5 & Acenaphtylene & POP & - & No & GC & 5.94 & 0.20 & Positive & $152.0 \rightarrow 151.0$ & 25 & $152.0 \rightarrow 126.0$ & 30 & 70 \\
\hline 6 & Acephate & I & $\begin{array}{l}\text { Not } \\
\text { approved }\end{array}$ & Yes & LC & 1.64 & 2.00 & Positive & $184.0 \rightarrow 143.0$ & 15 & $143.0 \rightarrow 95.0$ & 15 & 70 \\
\hline 7 & Acetaminophen (paracetamol) & V, NSAID & Approved & - & LC & 2.71 & 1.20 & Positive & $152.1 \rightarrow 65.0$ & 40 & $152.1 \rightarrow 93.0$ & 20 & 150 \\
\hline 8 & Acetamiprid & I & Approved & Yes & LC & 4.43 & 0.40 & Positive & $223.1 \rightarrow 126.0$ & 27 & $223.1 \rightarrow 90.0$ & 45 & 140 \\
\hline 9 & Acrinathrin & I, A & Approved & Yes & GC & 10.70 & 1.20 & Positive & $559.0 \rightarrow 208.0$ & 10 & $559.0 \rightarrow 181.0$ & 30 & 70 \\
\hline 10 & Albendazole & $\mathrm{V}, \mathrm{AH}$ & Approved & - & LC & 7.14 & 0.10 & Positive & $266.1 \rightarrow 234.1$ & 16 & $266.1 \rightarrow 191.0$ & 32 & 155 \\
\hline 11 & Aldicarb & I & $\begin{array}{l}\text { Not } \\
\text { approved }\end{array}$ & Yes & LC & 5.11 & 0.10 & Positive & $208.0 \rightarrow 116.0$ & 10 & $116.0 \rightarrow 89.1$ & 4 & 100 \\
\hline 12 & Aldicarb-sulfone & Met & - & Yes & LC & 3.21 & 0.40 & Positive & $240.1 \rightarrow 76.0$ & 16 & $223.1 \rightarrow 86.1$ & 13 & 75 \\
\hline 13 & Aldicarb-sulfoxide & Met & - & Yes & LC & 2.75 & 1.60 & Positive & $207.1 \rightarrow 131.9$ & 10 & $207.1 \rightarrow 89.1$ & 10 & 86 \\
\hline 14 & Aldrin & POP & - & Yes & GC & 9.90 & 0.40 & Positive & $255.0 \rightarrow 220.0$ & 25 & $263.0 \rightarrow 228.0$ & 10 & 70 \\
\hline 15 & Anthracene & POP & - & No & GC & 8.40 & 0.80 & Positive & $178.0 \rightarrow 176.0$ & 35 & $178.0 \rightarrow 152.0$ & 30 & 70 \\
\hline 16 & Atrazine & $\mathrm{H}$ & $\begin{array}{l}\text { Not } \\
\text { approved }\end{array}$ & No & LC & 6.73 & 0.10 & Positive & $216.0 \rightarrow 173.9$ & 15 & $216.0 \rightarrow 103.8$ & 30 & 130 \\
\hline 17 & Azinphos-methyl & I & $\begin{array}{l}\text { Not } \\
\text { approved }\end{array}$ & Yes & LC & 7.27 & 0.20 & Positive & $318.0 \rightarrow 132.1$ & 8 & $340.0 \rightarrow 160.0$ & 10 & 60 \\
\hline 18 & Azoxystrobin & $\mathrm{F}$ & Approved & Yes & LC & 7.59 & 0.10 & Positive & $404.1 \rightarrow 372.1$ & 8 & $404.1 \rightarrow 344.1$ & 24 & 110 \\
\hline 19 & BDE-28 & POP & - & No & GC & 12.22 & 0.20 & Positive & $406.0 \rightarrow 246.0$ & 20 & $406.0 \rightarrow 167.0$ & 25 & 70 \\
\hline 20 & BDE-47 & POP & - & No & GC & 14.31 & 0.20 & Positive & $326.0 \rightarrow 138.0$ & 45 & $484.0 \rightarrow 324.0$ & 25 & 70 \\
\hline 21 & BDE-85 & POP & - & No & GC & 17.08 & 0.10 & Positive & $564.0 \rightarrow 404.0$ & 25 & $566.0 \rightarrow 406.0$ & 25 & 70 \\
\hline 22 & BDE-99 & POP & - & No & GC & 16.27 & 0.10 & Positive & $566.0 \rightarrow 406.0$ & 25 & $564.0 \rightarrow 404.0$ & 30 & 70 \\
\hline 23 & BDE-100 & POP & - & No & GC & 15.85 & 0.10 & Positive & $566.0 \rightarrow 406.0$ & 25 & $564.0 \rightarrow 404.0$ & 25 & 70 \\
\hline 24 & BDE-153 & POP & - & No & GC & 18.04 & 0.20 & Positive & $644.0 \rightarrow 484.0$ & 25 & $486.0 \rightarrow 377.0$ & 30 & 70 \\
\hline 25 & BDE-154 & POP & - & No & GC & 17.47 & 0.10 & Positive & $644.0 \rightarrow 484.0$ & 25 & $486.0 \rightarrow 377.0$ & 30 & 70 \\
\hline 26 & BDE-183 & POP & - & No & GC & 20.12 & 0.20 & Positive & $561.6 \rightarrow 454.7$ & 40 & $563.6 \rightarrow 454.7$ & 40 & 70 \\
\hline 27 & Benalaxyl & $\mathrm{F}$ & Approved & No & LC & 8.96 & 0.10 & Positive & $326.2 \rightarrow 148.0$ & 20 & $326.2 \rightarrow 208.0$ & 12 & 90 \\
\hline 28 & Bendiocarb & I & $\begin{array}{l}\text { Not } \\
\text { approved }\end{array}$ & No & LC & 5.88 & 0.10 & Positive & $224.1 \rightarrow 166.9$ & 8 & $224.2 \rightarrow 108.9$ & 15 & 120 \\
\hline 29 & $\begin{array}{l}\text { Bendiocarb metabolite (2, 2-dimethylbenzo-1, 3-dioxol-4- } \\
\text { ol) }\end{array}$ & Met & - & No & GC & 4.84 & 1.20 & Positive & $166.0 \rightarrow 151.0$ & 10 & $166.0 \rightarrow 126.0$ & 20 & 70 \\
\hline 30 & Benfuracarb & $\mathrm{I}, \mathrm{AH}$ & $\begin{array}{l}\text { Not } \\
\text { approved }\end{array}$ & No & LC & 9.73 & 0.10 & Positive & $411.2 \rightarrow 190.0$ & 13 & $411.2 \rightarrow 252.0$ & 15 & 110 \\
\hline 31 & Benzo[a]anthracene & POP & - & No & GC & 13.95 & 0.80 & Positive & $228.0 \rightarrow 226.0$ & 40 & $228.0 \rightarrow 202.0$ & 35 & 70 \\
\hline 32 & Benzo[a]pyrene & POP & - & No & GC & 16.89 & 0.10 & Positive & $252.0 \rightarrow 250.0$ & 45 & $252.0 \rightarrow 248.0$ & 60 & 70 \\
\hline 33 & Benzo[b]fluoranthene & POP & - & No & GC & 16.30 & 0.80 & Positive & $252.0 \rightarrow 248.0$ & 60 & $252.0 \rightarrow 226.0$ & 35 & 70 \\
\hline 34 & Benzo[ghi]perylene & POP & - & No & GC & 19.61 & 0.40 & Positive & $276.0 \rightarrow 274.0$ & 50 & $276.0 \rightarrow 272.0$ & 60 & 70 \\
\hline 35 & Benzo[k]fluoranthene & POP & - & No & GC & 16.29 & 0.40 & Positive & $252.0 \rightarrow 250.0$ & 45 & $252.0 \rightarrow 224.0$ & 40 & 70 \\
\hline 36 & Bifenthrin & I & $\begin{array}{l}\text { Not } \\
\text { approved }\end{array}$ & Yes & GC & 11.25 & 0.20 & Positive & $440.0 \rightarrow 181.0$ & 5 & $440.0 \rightarrow 165.0$ & 60 & 94 \\
\hline 37 & Bitertanol & F & $\begin{array}{l}\text { Not } \\
\text { approved }\end{array}$ & Yes & LC & 9.23 & 0.40 & Positive & $338.2 \rightarrow 70.0$ & 4 & $338.2 \rightarrow 269.2$ & 5 & 100 \\
\hline 38 & Boscalid (formerly nicobifen) & $\mathrm{F}$ & Approved & Yes & GC & 7.84 & 0.10 & Positive & $3434.0 \rightarrow 272.0$ & 30 & $343.0 \rightarrow 140.0$ & 45 & 100 \\
\hline 39 & Brodifacoum & $\mathrm{R}$ & $\begin{array}{l}\text { Not } \\
\text { approved }\end{array}$ & No & LC & 10.78 & 0.80 & Negative & $521.3 \rightarrow 79.0$ & 50 & $523.3 \rightarrow 135.0$ & 45 & 220 \\
\hline 40 & Bromadiolone & $\mathrm{R}$ & Approved & No & LC & 9.75 & 0.40 & Negative & $525.3 \rightarrow 250.0$ & 40 & $527.3 \rightarrow 250.0$ & 40 & 200 \\
\hline 41 & Bromopropylate & A & Not & Yes & GC & 13.87 & 0.20 & Positive & $341.0 \rightarrow 183.0$ & 15 & $341.0 \rightarrow 157.0$ & 45 & 70 \\
\hline
\end{tabular}




\begin{tabular}{|c|c|c|c|c|c|c|c|c|c|c|c|c|c|}
\hline \multirow[t]{2}{*}{ No. } & \multirow[t]{2}{*}{ Compound } & \multirow[t]{2}{*}{ Category $^{\mathrm{a}}$} & \multirow{2}{*}{$\begin{array}{l}\text { Legal } \\
\text { status } \\
\text { in the } \mathrm{EU}^{\mathrm{b}}\end{array}$} & \multirow{2}{*}{$\begin{array}{l}\text { Subjected } \\
\text { to } \mathrm{MRL}^{\mathrm{c}}\end{array}$} & \multirow[t]{2}{*}{ Technique $^{\mathrm{d}}$} & \multirow[t]{2}{*}{ Retention time (min) } & \multirow{2}{*}{$\begin{array}{l}\mathrm{LOQ} \\
(\mathrm{ng} / \mathrm{ml})\end{array}$} & \multirow[t]{2}{*}{ Polarity } & \multicolumn{2}{|l|}{ Quantification } & \multicolumn{2}{|l|}{ Confirmation } & \multirow{2}{*}{$\begin{array}{l}\text { Fragmento } \\
\text { voltage (V) }\end{array}$} \\
\hline & & & & & & & & & $\begin{array}{l}\text { MRM transition } \\
(m / z)\end{array}$ & $\begin{array}{l}\text { Collision } \\
\text { energy } \\
(\mathrm{eV})\end{array}$ & $\begin{array}{l}\text { MRM } \\
\text { transition } \\
(m / z) \\
\end{array}$ & $\begin{array}{l}\text { Collision } \\
\text { energy } \\
(\mathrm{eV})\end{array}$ & \\
\hline & & & approved & & & & & & & & & & \\
\hline 42 & Bromuconazole (two isomers) & $\mathrm{F}$ & Approved & No & GC & $13.81 / 14.24$ & 0.20 & Positive & $295.0 \rightarrow 173.0$ & 10 & $295.0 \rightarrow 175.0$ & 10 & 70 \\
\hline 43 & Bupirimate & $\mathrm{F}$ & Approved & Yes & GC & 11.78 & 0.20 & Positive & $273.0 \rightarrow 108.0$ & 15 & $273.0 \rightarrow 193.0$ & 5 & 70 \\
\hline 44 & Buprofezin & I & Approved & Yes & LC & 9.83 & 0.10 & Positive & $306.1 \rightarrow 201.0$ & 12 & $306.1 \rightarrow 116.0$ & 12 & 140 \\
\hline 45 & Cadusafos (ebufos) & $\mathrm{I}, \mathrm{AH}$ & $\begin{array}{l}\text { Not } \\
\text { approved }\end{array}$ & No & LC & 9.39 & 0.10 & Positive & $271.1 \rightarrow 159.0$ & 16 & $271.1 \rightarrow 131.0$ & 22 & 100 \\
\hline 46 & Carbaryl & I & $\begin{array}{l}\text { Not } \\
\text { approved }\end{array}$ & Yes & LC & 6.21 & 0.10 & Positive & $202.1 \rightarrow 145.1$ & 4 & $202.1 \rightarrow 127.1$ & 28 & 95 \\
\hline 47 & Carbendazim (azole) & $\mathrm{F}$ & $\begin{array}{l}\text { Not } \\
\text { approved }\end{array}$ & Yes & LC & 2.90 & 0.40 & Positive & $192.1 \rightarrow 160.1$ & 4 & $202.1 \rightarrow 127.1$ & 28 & 90 \\
\hline 48 & Carbofuran & $\mathrm{I}, \mathrm{AH}$ & $\begin{array}{l}\text { Not } \\
\text { approved }\end{array}$ & Yes & LC & 5.91 & 0.10 & Positive & $222.1 \rightarrow 123.1$ & 20 & $222.1 \rightarrow 165.1$ & 30 & 80 \\
\hline 49 & Carbofuran-3-hydroxy & Met & - & Yes & LC & 4.27 & 0.40 & Positive & $238.1 \rightarrow 163.1$ & 10 & $238.1 \rightarrow 181.1$ & 10 & 110 \\
\hline 50 & Carbosulfan & $\mathrm{I}, \mathrm{AH}$ & $\begin{array}{l}\text { Not } \\
\text { approved }\end{array}$ & No & LC & 11.03 & 0.40 & Positive & $381.2 \rightarrow 160.2$ & 12 & $381.2 \rightarrow 76.1$ & 36 & 120 \\
\hline 51 & Cefuroxima axetil (two isomers) & $\mathrm{V}, \mathrm{MB}$ & $\begin{array}{l}\text { Not } \\
\text { approved }\end{array}$ & - & LC & 5.13 & 0.80 & Positive & $533.0 \rightarrow 447.0$ & 15 & $533.0 \rightarrow 386.0$ & 20 & 160 \\
\hline 52 & Chloramphenicol & $\mathrm{V}, \mathrm{MB}$ & Approved & - & LC & 4.63 & 2.00 & Negative & $321.0 \rightarrow 152.1$ & 4 & $323.0 \rightarrow 152.1$ & 4 & 113 \\
\hline 53 & Chlorantraniliprole & I & Approved & Yes & LC & 7.32 & 0.20 & Positive & $483.9 \rightarrow 452.9$ & 16 & $483.9 \rightarrow 285.9$ & 8 & 105 \\
\hline 54 & Chlorfenapyr & I, A & $\begin{array}{l}\text { Not } \\
\text { approved }\end{array}$ & Yes & GC & 12.01 & 1.20 & Positive & $247.0 \rightarrow 200.0$ & 30 & $247.0 \rightarrow 227.0$ & 15 & 70 \\
\hline 55 & Chlorfenvinphos & I & $\begin{array}{l}\text { Not } \\
\text { approved }\end{array}$ & No & LC & 9.09 & 0.20 & Positive & $361.1 \rightarrow 98.9$ & 34 & $358.9 \rightarrow 155.1$ & 8 & 105 \\
\hline 56 & Chlorobenzilate & A & $\begin{array}{l}\text { Not } \\
\text { approved }\end{array}$ & No & GC & 12.14 & 0.40 & Positive & $251.0 \rightarrow 111.0$ & 40 & $251.0 \rightarrow 139.0$ & 15 & 70 \\
\hline 57 & Chlorophacinone & $\mathrm{R}$ & $\begin{array}{l}\text { Not } \\
\text { approved }\end{array}$ & No & LC & 8.88 & 0.80 & Negative & $373.2 \rightarrow 201.0$ & 20 & $375.2 \rightarrow 203.0$ & 20 & 160 \\
\hline 58 & Chlorpropham & $\mathrm{H}$ & $\begin{array}{l}\text { Not } \\
\text { approved }\end{array}$ & Yes & GC & 7.13 & 0.20 & Positive & $213.0 \rightarrow 127.0$ & 15 & $153.0 \rightarrow 90.0$ & 25 & 70 \\
\hline 59 & Chlorpyrifos & I & $\begin{array}{l}\text { Not } \\
\text { approved }\end{array}$ & Yes & GC & 9.93 & 0.80 & Positive & $314.0 \rightarrow 258.0$ & 15 & $314.0 \rightarrow 286.0$ & 5 & 70 \\
\hline 60 & Chlorpyrifos methyl & I & $\begin{array}{l}\text { Not } \\
\text { approved }\end{array}$ & Yes & GC & 9.12 & 0.40 & Positive & $286.0 \rightarrow 93.0$ & 25 & $286.0 \rightarrow 271.0$ & 15 & 70 \\
\hline 61 & Chlorthal dimethyl & $\mathrm{H}$ & $\begin{array}{l}\text { Not } \\
\text { approved }\end{array}$ & No & GC & 10.02 & 0.20 & Positive & $300.9 \rightarrow 166.9$ & 55 & $300.9 \rightarrow 222.9$ & 25 & 70 \\
\hline 62 & Chrysene & POP & - & No & GC & 13.86 & 0.80 & Positive & $228.0 \rightarrow 226.0$ & 40 & $228.0 \rightarrow 227.0$ & 25 & 70 \\
\hline 63 & Clindamycin & $\mathrm{V}, \mathrm{MB}$ & Approved & - & LC & 5.33 & 0.40 & Positive & $425.2 \rightarrow 126.1$ & 20 & $425.2 \rightarrow 377.2$ & 20 & 150 \\
\hline 64 & Clofentezine & A & Approved & Yes & LC & 9.19 & 0.40 & Positive & $303.1 \rightarrow 138.0$ & 12 & $303.1 \rightarrow 102.0$ & 40 & 120 \\
\hline 65 & Clothianidin & I & $\begin{array}{l}\text { Not } \\
\text { approved }\end{array}$ & Yes & LC & 3.91 & 1.20 & Positive & $250.0 \rightarrow 169.0$ & 8 & $250.0 \rightarrow 131.9$ & 8 & 100 \\
\hline 66 & Cloxacillin & $\mathrm{V}, \mathrm{MB}$ & Approved & - & LC & 6.86 & 1.60 & Positive & $436.1 \rightarrow 160.0$ & 8 & $436.1 \rightarrow 277.0$ & 12 & 126 \\
\hline 67 & Cortiscosterone & $\mathrm{V}, \mathrm{GC}$ & $\begin{array}{l}\text { Not } \\
\text { approved }\end{array}$ & - & LC & 7.89 & 0.80 & Positive & $389.1 \rightarrow 329.0$ & 13 & $389.1 \rightarrow 371.0$ & 13 & 80 \\
\hline 68 & Coumachlor & $\mathrm{R}$ & $\begin{array}{l}\text { Not } \\
\text { approved }\end{array}$ & No & LC & 8.63 & 0.20 & Positive & $343.1 \rightarrow 162.8$ & 15 & $342.1 \rightarrow 285.0$ & 15 & 120 \\
\hline 69 & Coumaphos & I, A & $\begin{array}{l}\text { Not } \\
\text { approved }\end{array}$ & No & LC & 8.98 & 0.10 & Positive & $363.0 \rightarrow 227.0$ & 30 & $363.0 \rightarrow 306.9$ & 15 & 120 \\
\hline 70 & Coumatetralyl & $\mathrm{R}$ & $\begin{array}{l}\text { Not } \\
\text { approved }\end{array}$ & No & LC & 8.31 & 0.40 & Negative & $291.1 \rightarrow 141.0$ & 30 & $291.1 \rightarrow 247.0$ & 20 & 140 \\
\hline 71 & Cyazofamid & $\mathrm{F}$ & Approved & Yes & LC & 8.49 & 0.80 & Positive & $325.0 \rightarrow 108.0$ & 20 & $325.0 \rightarrow 261.1$ & 15 & 90 \\
\hline 72 & Cyflufenamid & $\mathrm{F}$ & Approved & Yes & LC & 9.18 & 0.20 & Positive & $413.1 \rightarrow 223.1$ & 33 & $413.1 \rightarrow 295.1$ & 23 & 70 \\
\hline 73 & Cyfluthrin (sum of four isomers) & I & Not & Yes & GC & 16.07/16.19/16.25/16.32 & 1.20 & Positive & $226.0 \rightarrow 206.0$ & 25 & $198.9 \rightarrow 170.1$ & 25 & 70 \\
\hline
\end{tabular}




\begin{tabular}{|c|c|c|c|c|c|c|c|c|c|c|c|c|c|}
\hline 74 & Cyhalothrin (lambda isomer) & I & Approved & Yes & LC & 10.49 & 2.00 & Positive & $467.0 \rightarrow 225.0$ & 10 & $467.0 \rightarrow 141.0$ & 46 & 66 \\
\hline 75 & Cymoxanil & $\mathrm{F}$ & Approved & Yes & LC & 4.67 & 0.40 & Positive & $199.1 \rightarrow 128.0$ & 4 & $199.1 \rightarrow 110.9$ & 12 & 90 \\
\hline 76 & Cypermethrin (sum of four isomers) & I & Approved $^{\mathrm{f}}$ & Yes & GC & $16.34 / 16.44 / 16.52 / 16.63$ & 4.00 & Positive & $163.0 \rightarrow 109.0$ & 20 & $163.0 \rightarrow 127.0$ & 5 & 70 \\
\hline 77 & Cyproconazole (two isomers) & $\mathrm{F}$ & Approved & Yes & LC & 8.14 & 0.40 & Positive & $292.2 \rightarrow 70.2$ & 18 & $292.2 \rightarrow 125.1$ & 24 & 100 \\
\hline 78 & Cyprodinil & $\mathrm{F}$ & Approved & Yes & LC & 8.46 & 0.20 & Positive & $226.0 \rightarrow 93.0$ & 33 & $226.0 \rightarrow 108$ & 25 & 100 \\
\hline 79 & Cyromazine & I, A & $\begin{array}{l}\text { Not } \\
\text { approved }\end{array}$ & Yes & LC & 1.23 & 2.00 & Positive & $167.1 \rightarrow 85.0$ & 16 & $167.1 \rightarrow 125.0$ & 20 & 120 \\
\hline 80 & Danofloxacin & $\mathrm{V}, \mathrm{MB}$ & Approved & - & LC & 4.04 & 1.20 & Positive & $358.2 \rightarrow 340.1$ & 20 & $358.2 \rightarrow 82.1$ & 50 & 159 \\
\hline 81 & Dazomet & $\begin{array}{l}\text { I, A, AH, } \\
\text { F, H }\end{array}$ & Approved & No & GC & 7.80 & 1.60 & Positive & $161.9 \rightarrow 44.0$ & 28 & $161.9 \rightarrow 89.0$ & 5 & 70 \\
\hline 82 & Deltamethrin & $\mathrm{I}, \mathrm{A}$ & Approved & Yes & LC & 10.65 & 0.80 & Positive & $523.0 \rightarrow 281.0$ & 10 & $523.0 \rightarrow 506.0$ & 5 & 100 \\
\hline 83 & Demeton-S-methyl & $\mathrm{I}, \mathrm{A}$ & $\begin{array}{l}\text { Not } \\
\text { approved }\end{array}$ & No & LC & 5.97 & 0.10 & Positive & $230.9 \rightarrow 88.9$ & 5 & $230.9 \rightarrow 61.0$ & 30 & 50 \\
\hline 84 & Demeton-S-methyl-sulfone (Dioxydemeton) & I, A & $\begin{array}{l}\text { Not } \\
\text { approved }\end{array}$ & No & LC & 3.31 & 0.40 & Positive & $263.0 \rightarrow 169.0$ & 24 & $263.0 \rightarrow 109.0$ & 12 & 120 \\
\hline 85 & Dexamethasone & V, GC & Approved & - & LC & 7.16 & 0.40 & Positive & $393.2 \rightarrow 373.2$ & 2 & $393.2 \rightarrow 355.2$ & 6 & 103 \\
\hline 86 & Diazinon & I & $\begin{array}{l}\text { Not } \\
\text { approved }\end{array}$ & Yes & GC & 8.29 & 0.40 & Positive & $137.1 \rightarrow 54.0$ & 20 & $304.0 \rightarrow 179.0$ & 15 & 70 \\
\hline 87 & Dibenzo $[a, h]$ anthracene & РОР & - & No & GC & 19.15 & 0.40 & Positive & $278.0 \rightarrow 276.0$ & 40 & $278.0 \rightarrow 250.0$ & 60 & 70 \\
\hline 88 & Dichlorodiphenyldichloroethane ( $\mathrm{p}, \mathrm{p}^{\prime}$ DDD) & POP & - & Yes & GC & 12.31 & 0.10 & Positive & $235.0 \rightarrow 165.0$ & 20 & $235.0 \rightarrow 199.0$ & 15 & 70 \\
\hline 89 & Dichlorodiphenyldichloroethylene ( $\mathrm{p}, \mathrm{p}^{\prime}$ DDE) & POP & - & Yes & GC & 11.58 & 0.10 & Positive & $318.0 \rightarrow 176.0$ & 60 & $318.0 \rightarrow 248.0$ & 30 & 70 \\
\hline 90 & Dichlorodiphenyltrichloroethane ( $\mathrm{p}, \mathrm{p}^{\prime}$ DDT) & POP & - & Yes & GC & 12.84 & 1.20 & Positive & $235.0 \rightarrow 165.0$ & 40 & $235.0 \rightarrow 199.0$ & 15 & 70 \\
\hline 91 & Diclofenac & V, NSAID & Approved & - & LC & 8.73 & 0.80 & Positive & $296.0 \rightarrow 215.1$ & 16 & $296.0 \rightarrow 214.1$ & 48 & 103 \\
\hline 92 & Dicloran & $\begin{array}{l}\text { F, MB, } \\
\text { WP }\end{array}$ & $\begin{array}{l}\text { Not } \\
\text { approved }\end{array}$ & Yes & GC & 7.80 & 0.10 & Positive & $206.0 \rightarrow 176.0$ & 10 & $206.0 \rightarrow 148.0$ & 25 & 70 \\
\hline 93 & Dicloxacillin & $\mathrm{V}, \mathrm{MB}$ & $\begin{array}{l}\text { Not } \\
\text { approved }\end{array}$ & - & LC & 7.24 & 1.20 & Positive & $470.0 \rightarrow 160.0$ & 8 & $470.0 \rightarrow 310.8$ & 10 & 106 \\
\hline 94 & Dieldrin & POP & - & Yes & GC & 11.66 & 1.20 & Positive & $263.0 \rightarrow 228.0$ & 15 & $277.0 \rightarrow 241.0$ & 15 & 70 \\
\hline 95 & Diethathyl ethyl & $\mathrm{H}$ & $\begin{array}{l}\text { Not } \\
\text { approved }\end{array}$ & No & LC & 8.71 & 0.20 & Positive & $312.2 \rightarrow 238.1$ & 15 & $312.2 \rightarrow 162.0$ & 30 & 120 \\
\hline 96 & Diethofencarb & $\begin{array}{l}\text { F, MB, } \\
\text { WP }\end{array}$ & Approved & Yes & LC & 7.57 & 0.10 & Positive & $268.2 \rightarrow 226.1$ & 5 & $268.2 \rightarrow 152.0$ & 20 & 110 \\
\hline 97 & Difenacoum & $\mathrm{R}$ & $\begin{array}{l}\text { Not } \\
\text { approved }\end{array}$ & No & LC & 10.38 & 0.40 & Negative & $443.2 \rightarrow 135.0$ & 40 & $443.2 \rightarrow 293.0$ & 35 & 200 \\
\hline 98 & Difenoconazole & $\begin{array}{l}\text { F, MB, } \\
\text { WP }\end{array}$ & Approved & Yes & LC & 9.41 & 0.40 & Positive & $406.1 \rightarrow 250.9$ & 28 & $406.1 \rightarrow 337.0$ & 16 & 176 \\
\hline 99 & Difethialone & $\mathrm{R}$ & $\begin{array}{l}\text { Not } \\
\text { approved }\end{array}$ & No & LC & 10.93 & 0.80 & Negative & $537.3 \rightarrow 79.0$ & 50 & $537.3 \rightarrow 151.0$ & 45 & 220 \\
\hline 100 & Difloxacin & $\mathrm{V}, \mathrm{MB}$ & $\begin{array}{l}\text { Not } \\
\text { approved }\end{array}$ & - & LC & 3.86 & 0.80 & Positive & $400.2 \rightarrow 382.1$ & 20 & $400.2 \rightarrow 356.1$ & 16 & 149 \\
\hline 101 & Diflubenzuron & I & Approved & Yes & LC & 8.63 & 1.20 & Positive & $311.0 \rightarrow 158.0$ & 8 & $311.0 \rightarrow 141.0$ & 32 & 90 \\
\hline 102 & Diflufenican & $\mathrm{H}$ & Approved & No & LC & 9.51 & 0.10 & Positive & $395.1 \rightarrow 266.0$ & 24 & $395.1 \rightarrow 246.0$ & 36 & 150 \\
\hline 103 & Dimethenamid-P (and its R-isomer) & $\mathrm{H}$ & Approved & No & LC & 7.68 & 0.10 & Positive & $276.1 \rightarrow 244.1$ & 10 & $276.1 \rightarrow 168.1$ & 20 & 125 \\
\hline 104 & Dimethoate & I & $\begin{array}{l}\text { Not } \\
\text { approved }\end{array}$ & Yes & LC & 4.21 & 0.40 & Positive & $230.0 \rightarrow 125.0$ & 16 & $230.0 \rightarrow 198.8$ & 20 & 70 \\
\hline 105 & Dimethomorph (two isomers) & $\begin{array}{l}\text { F, MB, } \\
\text { WP }\end{array}$ & Approved & Yes & LC & 7.86 & 0.40 & Positive & $388.1 \rightarrow 301.1$ & 20 & $388.1 \rightarrow 165.1$ & 32 & 180 \\
\hline 106 & $\begin{array}{l}\text { Dimethylphenylsulfamide (DMSA, metabolite of } \\
\text { dichlofluanid) }\end{array}$ & $\mathrm{Met}^{\mathrm{h}}$ & - & No & LC & 5.21 & 0.80 & Positive & $201.1 \rightarrow 92.1$ & 15 & $201.1 \rightarrow 137.1$ & 5 & 100 \\
\hline 107 & Diniconazole-M & $\begin{array}{l}\text { F, MB, } \\
\text { WP }\end{array}$ & $\begin{array}{l}\text { Not } \\
\text { approved }\end{array}$ & Yes & LC & 9.34 & 0.20 & Positive & $326.1 \rightarrow 70.0$ & 28 & $328.1 \rightarrow 70.0$ & 28 & 110 \\
\hline 108 & Dinocap & $\begin{array}{l}\text { F, MB, } \\
\text { WP }\end{array}$ & $\begin{array}{l}\text { Not } \\
\text { approved }\end{array}$ & No & LC & 10.51 & 0.80 & Negative & $295.4 \rightarrow 208.9$ & 30 & $295.4 \rightarrow 193.0$ & 35 & 150 \\
\hline 109 & Diphacinone & $\mathrm{R}$ & $\begin{array}{l}\text { Not } \\
\text { approved }\end{array}$ & No & LC & 8.60 & 1.20 & Negative & $339.1 \rightarrow 167.0$ & 25 & $339.1 \rightarrow 145.0$ & 20 & 170 \\
\hline 110 & Diphenylamine & PHP & $\begin{array}{l}\text { Not } \\
\text { approved }\end{array}$ & Yes & GC & 6.98 & 0.20 & Positive & $168.0 \rightarrow 167.2$ & 15 & $169.0 \rightarrow 66.0$ & 15 & 70 \\
\hline 111 & Dodine & $\begin{array}{l}\text { F, MB, } \\
\text { WP }\end{array}$ & Approved & Yes & LC & 9.02 & 0.40 & Positive & $228.3 \rightarrow 43.0$ & 40 & $228.3 \rightarrow 57.0$ & 25 & 150 \\
\hline
\end{tabular}




\begin{tabular}{|c|c|c|c|c|c|c|c|c|c|c|c|c|c|}
\hline \multirow[t]{2}{*}{ No. } & \multirow[t]{2}{*}{ Compound } & \multirow[t]{2}{*}{ Category $^{\mathrm{a}}$} & \multirow{2}{*}{$\begin{array}{l}\text { Legal } \\
\text { status } \\
\text { in the } \mathrm{EU}^{\mathrm{b}}\end{array}$} & \multirow{2}{*}{$\begin{array}{l}\text { Subjected } \\
\text { to } \text { MRL }^{\mathrm{c}}\end{array}$} & \multirow[t]{2}{*}{ Technique $^{d}$} & \multirow[t]{2}{*}{ Retention time (min) } & \multirow{2}{*}{$\begin{array}{l}\text { LOQ } \\
(\mathrm{ng} / \mathrm{ml})\end{array}$} & \multirow[t]{2}{*}{ Polarity } & \multicolumn{2}{|l|}{ Quantification } & \multicolumn{2}{|l|}{ Confirmation } & \multirow{2}{*}{$\begin{array}{l}\text { Fragmentor } \\
\text { voltage (V) }\end{array}$} \\
\hline & & & & & & & & & $\begin{array}{l}\text { MRM transition } \\
(m / z)\end{array}$ & $\begin{array}{l}\text { Collision } \\
\text { energy } \\
(\mathrm{eV})\end{array}$ & $\begin{array}{l}\text { MRM } \\
\text { transition } \\
(m / z)\end{array}$ & $\begin{array}{l}\text { Collision } \\
\text { energy } \\
(\mathrm{eV})\end{array}$ & \\
\hline 112 & Endosulfan alfa & POP & - & Yes & GC & 11.21 & 0.80 & Positive & $241.0 \rightarrow 206.0$ & 15 & $195.0 \rightarrow 160.0$ & 10 & 70 \\
\hline 113 & Endosulfan beta & POP & - & Yes & GC & 12.21 & 0.80 & Positive & $241.0 \rightarrow 206.0$ & 15 & $195.0 \rightarrow 159.0$ & 15 & 70 \\
\hline 114 & Endosulfan sulfate & POP & - & No & GC & 12.96 & 0.80 & Positive & $270.0 \rightarrow 235.0$ & 15 & $387.0 \rightarrow 289.0$ & 5 & 70 \\
\hline 115 & Endrin & POP & - & No & GC & 12.05 & 1.60 & Positive & $263.0 \rightarrow 193.0$ & 35 & $245.0 \rightarrow 173.0$ & 25 & 70 \\
\hline 116 & Enrofloxacin & $\mathrm{V}, \mathrm{MB}$ & Approved & - & LC & 3.94 & 1.20 & Positive & $360.2 \rightarrow 316.1$ & 16 & $360.2 \rightarrow 245.1$ & 28 & 144 \\
\hline 117 & EPN & I, A & $\begin{array}{l}\text { Not } \\
\text { approved }\end{array}$ & No & GC & 13.90 & 0.80 & Positive & $157.0 \rightarrow 63.0$ & 10 & $157.0 \rightarrow 110.0$ & 15 & 70 \\
\hline 118 & Epoxiconazole & $\mathrm{F}$ & Approved & Yes & LC & 8.47 & 0.20 & Positive & $330.0 \rightarrow 120.9$ & 24 & $330.1 \rightarrow 100.9$ & 50 & 120 \\
\hline 119 & Eprinomectin & $\mathrm{V}, \mathrm{MB}$ & Approved & - & LC & 10.84 & 0.20 & Positive & $878.5 \rightarrow 186.0$ & 15 & $936.5 \rightarrow 490.4$ & 60 & 160 \\
\hline 120 & Eritromicin & $\mathrm{V}, \mathrm{MB}$ & Approved & - & LC & 6.74 & 0.20 & Positive & $734.5 \rightarrow 158.1$ & 32 & $734.5 \rightarrow 576.3$ & 16 & 172 \\
\hline 121 & Esfenvalerate & I & Approved & No & GC & 17.56 & 2.00 & Positive & $167.1 \rightarrow 125.1$ & 15 & $167.1 \rightarrow 89.1$ & 45 & 70 \\
\hline 122 & Ethion (diethion) & I, A & $\begin{array}{l}\text { Not } \\
\text { approved }\end{array}$ & Yes & $\mathrm{LC}$ & 10.03 & 0.10 & Positive & $385.0 \rightarrow 199.0$ & 5 & $385.0 \rightarrow 171.0$ & 10 & 100 \\
\hline 123 & Ethirimol & $\begin{array}{l}\text { F, MB, } \\
\text { WP }\end{array}$ & $\begin{array}{l}\text { Not } \\
\text { approved }\end{array}$ & Yes & LC & 4.80 & 0.40 & Positive & $210.2 \rightarrow 140.1$ & 20 & $210.2 \rightarrow 98.1$ & 28 & 160 \\
\hline 124 & Ethofumesate & $\mathrm{H}$ & Approved & No & GC & 9.59 & 0.80 & Positive & $286.0 \rightarrow 207.0$ & 5 & $286.0 \rightarrow 161.0$ & 20 & 70 \\
\hline 125 & Ethoprophos & $\mathrm{I}, \mathrm{AH}$ & $\begin{array}{l}\text { Not } \\
\text { approved }\end{array}$ & No & LC & 8.38 & 0.20 & Positive & $243.1 \rightarrow 97.0$ & 30 & $243.1 \rightarrow 130.9$ & 15 & 90 \\
\hline 126 & Etofenprox & I, A & Approved & Yes & GC & 16.75 & 0.80 & Positive & $163.0 \rightarrow 107.0$ & 20 & $163.0 \rightarrow 135.0$ & 10 & 70 \\
\hline 127 & Etoxazole & A & Approved & Yes & LC & 10.34 & 0.10 & Positive & $360.1 \rightarrow 141.0$ & 26 & $360.1 \rightarrow 304.0$ & 16 & 160 \\
\hline 128 & Famoxadone & $\mathrm{H}$ & Approved & Yes & LC & 9.07 & 1.20 & Positive & $392.1 \rightarrow 330.9$ & 5 & $392.2 \rightarrow 238.1$ & 12 & 110 \\
\hline 129 & Fenamidone & $\mathrm{F}$ & $\begin{array}{l}\text { Not } \\
\text { approved }\end{array}$ & Yes & LC & 9.06 & 0.10 & Positive & $392.1 \rightarrow 330.9$ & 5 & $392.1 \rightarrow 238.1$ & 12 & 110 \\
\hline 130 & Fenamiphos & $\mathrm{I}, \mathrm{AH}$ & Approved & Yes & LC & 7.72 & 0.10 & Positive & $304.1 \rightarrow 217.1$ & 20 & $304.1 \rightarrow 202.0$ & 36 & 120 \\
\hline 131 & Fenamiphos sulfone & Met & - & Yes & LC & 8.63 & 0.20 & Positive & $336.1 \rightarrow 188.0$ & 31 & $336.1 \rightarrow 266.0$ & 23 & 120 \\
\hline 132 & Fenamiphos sulfoxide & Met & - & Yes & LC & 5.93 & 0.40 & Positive & $320.1 \rightarrow 233.0$ & 20 & $320.1 \rightarrow 108.1$ & 44 & 120 \\
\hline 133 & Fenarimol & $\begin{array}{l}\text { F, MB, } \\
\text { WP }\end{array}$ & $\begin{array}{l}\text { Not } \\
\text { approved }\end{array}$ & Yes & GC & 15.03 & 0.20 & Positive & $139.0 \rightarrow 75.0$ & 30 & $139.0 \rightarrow 111.0$ & 15 & 70 \\
\hline 134 & Fenazaquin & A & Approved & Yes & LC & 10.73 & 0.80 & Positive & $307.2 \rightarrow 57.1$ & 25 & $307.2 \rightarrow 161.1$ & 16 & 90 \\
\hline 135 & Fenbendazole & $\mathrm{V}, \mathrm{AH}$ & Approved & - & LC & 8.04 & 0.10 & Positive & $300.1 \rightarrow 268.1$ & 20 & $300.1 \rightarrow 159.0$ & 36 & 156 \\
\hline 136 & Fenbuconazole & $\mathrm{F}, \mathrm{V}$ & Approved & Yes & GC & 16.17 & 0.40 & Positive & $198.0 \rightarrow 102.0$ & 30 & $198.0 \rightarrow 78.0$ & 30 & 70 \\
\hline 137 & Fenbutatin oxide & I, A & $\begin{array}{l}\text { Not } \\
\text { approved }\end{array}$ & Yes & LC & 11.67 & 0.80 & Positive & $519.0 \rightarrow 197.0$ & 55 & $517.3 \rightarrow 194.9$ & 60 & 180 \\
\hline 138 & Fenhexamid & $\mathrm{F}$ & Approved & Yes & LC & 8.35 & 1.60 & Positive & $302.1 \rightarrow 97.1$ & 20 & $302.1 \rightarrow 55.1$ & 40 & 130 \\
\hline 139 & Fenitrothion & I & $\begin{array}{l}\text { Not } \\
\text { approved }\end{array}$ & Yes & GC & 9.57 & 0.20 & Positive & $277.0 \rightarrow 109.0$ & 15 & $277.0 \rightarrow 125.0$ & 15 & 70 \\
\hline 140 & Fenoxycarb & I & Approved & Yes & LC & 8.69 & 0.10 & Positive & $302.1 \rightarrow 88.0$ & 20 & $302.1 \rightarrow 116.1$ & 10 & 110 \\
\hline 141 & Fenpropathrin & I, A & $\begin{array}{l}\text { Not } \\
\text { approved }\end{array}$ & Yes & LC & 10.43 & 0.40 & Positive & $367.2 \rightarrow 125.0$ & 16 & $350.1 \rightarrow 125.0$ & 16 & 72 \\
\hline 142 & Fenpropidin & $\mathrm{F}$ & Approved & Yes & LC & 7.13 & 0.10 & Positive & $274.3 \rightarrow 147.0$ & 30 & $274.3 \rightarrow 86.0$ & 25 & 170 \\
\hline 143 & Fenpropimorph & $\mathrm{F}$ & $\begin{array}{l}\text { Not } \\
\text { approved }\end{array}$ & Yes & LC & 7.37 & 0.10 & Positive & $304.3 \rightarrow 147.1$ & 30 & $304.3 \rightarrow 130.0$ & 25 & 120 \\
\hline 144 & Fenpyroximate & A & Approved & Yes & LC & 10.49 & 0.40 & Positive & $422.2 \rightarrow 366.2$ & 12 & $422.2 \rightarrow 135.0$ & 36 & 160 \\
\hline 145 & Fenthion & I, A & $\begin{array}{l}\text { Not } \\
\text { approved }\end{array}$ & Yes & $\mathrm{LC}$ & 8.90 & 0.10 & Positive & $278.9 \rightarrow 168.8$ & 18 & $278.9 \rightarrow 247.0$ & 8 & 98 \\
\hline 146 & Fenthion oxon & Met & - & Yes & LC & 7.31 & 0.10 & Positive & $263.1 \rightarrow 231.2$ & 16 & $263.1 \rightarrow 216.0$ & 24 & 120 \\
\hline 147 & Fenthion oxon sulfone & Met & - & Yes & LC & 4.50 & 0.80 & Positive & $295.0 \rightarrow 217.0$ & 15 & $295.0 \rightarrow 104.2$ & 24 & 110 \\
\hline 148 & Fenthion oxon sulfoxide & Met & - & Yes & LC & 4.26 & 0.20 & Positive & $279.0 \rightarrow 264.2$ & 20 & $279.0 \rightarrow 104.1$ & 28 & 110 \\
\hline 149 & Fenthion sulfone & Met & - & Yes & LC & 6.39 & 0.80 & Positive & $311.0 \rightarrow 125.0$ & 22 & $311.0 \rightarrow 109.0$ & 28 & 140 \\
\hline 150 & Fenthion sulfoxide & Met & - & Yes & LC & 6.16 & 0.40 & Positive & $295.0 \rightarrow 108.9$ & 30 & $295.0 \rightarrow 280.0$ & 18 & 140 \\
\hline 151 & Fenvalerate & I & $\begin{array}{l}\text { Not } \\
\text { approved }\end{array}$ & Yes & GC & 17.36 & 2.00 & Positive & $167.0 \rightarrow 125.1$ & 22 & $167.0 \rightarrow 89.0$ & 30 & 70 \\
\hline 152 & Fipronil & $\mathrm{I}, \mathrm{V}$ & Not & Yes & LC & 8.68 & 0.20 & Negative & $435.0 \rightarrow 330.0$ & 12 & $435.0 \rightarrow 249.9$ & 26 & 116 \\
\hline
\end{tabular}




\begin{tabular}{|c|c|c|c|c|c|c|c|c|c|c|c|c|c|}
\hline & & & approved & & & & & & & & & & \\
\hline 153 & Fipronil sulfide & Met & - & Yes & GC & 10.49 & 0.80 & Positive & $351.0 \rightarrow 255.0$ & 20 & $420.0 \rightarrow 351.0$ & 25 & 70 \\
\hline 154 & Flocoumafen & $\mathrm{R}$ & $\begin{array}{l}\text { Not } \\
\text { approved }\end{array}$ & No & $\mathrm{LC}$ & 10.44 & 0.20 & Negative & $541.3 \rightarrow 382.0$ & 25 & $541.3 \rightarrow 161.0$ & 40 & 230 \\
\hline 155 & Fluazinam & $\mathrm{F}$ & Approved & No & LC & 10.01 & 0.20 & Negative & $462.9 \rightarrow 416.0$ & 10 & $462.9 \rightarrow 398.0$ & 9 & 140 \\
\hline 156 & Flubendiamide & I & Approved & Yes & LC & 8.82 & 2.00 & Positive & $408.0 \rightarrow 274.0$ & 15 & $408.0 \rightarrow 256.0$ & 30 & 120 \\
\hline 157 & Flucythrinate (two isomers) & $\mathrm{I}, \mathrm{A}$ & $\begin{array}{l}\text { Not } \\
\text { approved }\end{array}$ & No & GC & $16.67 / 16.84$ & 0.80 & Positive & $156.9 \rightarrow 107.1$ & 15 & $199.1 \rightarrow 107.1$ & 25 & 70 \\
\hline 158 & Fludioxonil & $\mathrm{F}$ & Approved & Yes & GC & 11.51 & 0.20 & Positive & $248.0 \rightarrow 127.0$ & 30 & $248.1 \rightarrow 182.1$ & 10 & 70 \\
\hline 159 & Flufenoxuron & $\mathrm{I}, \mathrm{A}$ & $\begin{array}{l}\text { Not } \\
\text { approved }\end{array}$ & Yes & LC & 10.37 & 0.10 & Positive & $489.1 \rightarrow 158.0$ & 20 & $489.1 \rightarrow 140.9$ & 56 & 110 \\
\hline 160 & Flumequine & $\mathrm{V}, \mathrm{MB}$ & Approved & - & LC & 6.12 & 0.10 & Positive & $262.1 \rightarrow 244.0$ & 16 & $262.1 \rightarrow 202.0$ & 32 & 116 \\
\hline 161 & Flunixin & $\mathrm{V}$, NSAID & Approved & - & LC & 8.09 & 0.20 & Positive & $297.1 \rightarrow 279.1$ & 24 & $297.1 \rightarrow 264.1$ & 32 & 141 \\
\hline 162 & Fluopyram & $\mathrm{F}$ & Approved & Yes & GC & 10.61 & 0.20 & Positive & $173.0 \rightarrow 95$ & 35 & $223.0 \rightarrow 196.0$ & 40 & 70 \\
\hline 163 & Fluoranthene & POP & - & No & GC & 10.66 & 0.20 & Positive & $202.0 \rightarrow 201.0$ & 27 & $202.0 \rightarrow 152.0$ & 42 & 70 \\
\hline 164 & Fluorene & POP & - & No & GC & 6.81 & 0.20 & Positive & $165.0 \rightarrow 163.0$ & 40 & $165.0 \rightarrow 139.0$ & 30 & 70 \\
\hline 165 & Fluquinconazole & $\mathrm{F}$ & Approved & Yes & GC & 15.81 & 0.20 & Positive & $340.0 \rightarrow 298.0$ & 15 & $340.0 \rightarrow 286.0$ & 25 & 70 \\
\hline 166 & Flusilazole & $\begin{array}{l}\text { F, MB, } \\
\text { WP }\end{array}$ & $\begin{array}{l}\text { Not } \\
\text { approved }\end{array}$ & Yes & LC & 8.64 & 0.20 & Positive & $316.1 \rightarrow 247.1$ & 15 & $316.1 \rightarrow 165.0$ & 20 & 160 \\
\hline 167 & Flutolanil & $\begin{array}{l}\mathrm{F}, \mathrm{MB} \text {, } \\
\text { WP }\end{array}$ & Approved & No & LC & 7.93 & 0.10 & Positive & $324.1 \rightarrow 262.1$ & 16 & $324.1 \rightarrow 242.1$ & 24 & 130 \\
\hline 168 & Flutriafol & $\mathrm{F}$ & Approved & Yes & GC & 11.26 & 0.20 & Positive & $219.0 \rightarrow 95.0$ & 35 & $219.0 \rightarrow 123.0$ & 15 & 70 \\
\hline 169 & Fluvalinate tau & I, A & Approved & Yes & GC & 17.56 & 4.00 & Positive & $250.1 \rightarrow 55.1$ & 30 & $252.0 \rightarrow 200.0$ & 20 & 70 \\
\hline 170 & Fonofos & I & $\begin{array}{l}\text { Not } \\
\text { approved }\end{array}$ & No & GC & 8.24 & 0.40 & Positive & $246.0 \rightarrow 109.0$ & 15 & $246.0 \rightarrow 237.0$ & 5 & 70 \\
\hline 171 & Formetanate & I, A & Approved & Yes & LC & 1.76 & 0.10 & Positive & $222.1 \rightarrow 165.1$ & 12 & $222.1 \rightarrow 46.2$ & 28 & 105 \\
\hline 172 & Fosthiazate & $\mathrm{AH}, \mathrm{V}$ & Approved & Yes & LC & 6.50 & 0.10 & Positive & $284.0 \rightarrow 104.0$ & 20 & $284.0 \rightarrow 227.8$ & 8 & 90 \\
\hline 173 & Heptachlor & POP & - & Yes & GC & 9.31 & 0.80 & Positive & $272.0 \rightarrow 237.0$ & 15 & $274.0 \rightarrow 239.0$ & 15 & 70 \\
\hline 174 & Hexachlorobencene & POP & - & Yes & GC & 7.77 & 0.20 & Positive & $284.0 \rightarrow 214.0$ & 40 & $284.0 \rightarrow 249.0$ & 25 & 70 \\
\hline 175 & Hexachlorocyclohexane (alpha) & POP & - & Yes & GC & 7.64 & 0.40 & Positive & $219.0 \rightarrow 109.0$ & 10 & $219.0 \rightarrow 183.0$ & 10 & 70 \\
\hline 176 & Hexachlorocyclohexane (beta) & POP & - & Yes & GC & 8.02 & 0.40 & Positive & $219.0 \rightarrow 109.0$ & 40 & $219.0 \rightarrow 183.0$ & 5 & 70 \\
\hline 177 & Hexachlorocyclohexane (delta) & POP & - & No & GC & 8.50 & 0.20 & Positive & $219.0 \rightarrow 109.0$ & 45 & $219.0 \rightarrow 183.0$ & 5 & 70 \\
\hline 178 & Hexaclorocyclohexane (gamma, lindane) & POP & - & Yes & GC & 8.13 & 1.20 & Positive & $291.0 \rightarrow 109.0$ & 40 & $219.0 \rightarrow 183.0$ & 10 & 70 \\
\hline 179 & Hexaconazole (two isomers) & $\begin{array}{l}\text { F, MB, } \\
\text { WP }\end{array}$ & $\begin{array}{l}\text { Not } \\
\text { approved }\end{array}$ & Yes & $\mathrm{LC}$ & 8.49 & 0.80 & Positive & $314.1 \rightarrow 70.1$ & 20 & $316.0 \rightarrow 70.1$ & 20 & 95 \\
\hline 180 & Hexaflumuron & I & $\begin{array}{l}\text { Not } \\
\text { approved }\end{array}$ & No & $\mathrm{LC}$ & 9.58 & 0.40 & Negative & $458.8 \rightarrow 439.0$ & 8 & $458.8 \rightarrow 175.0$ & 30 & 100 \\
\hline 181 & Hexythiazox & A & Approved & Yes & LC & 10.18 & 0.10 & Positive & $353.1 \rightarrow 227.9$ & 8 & $353.1 \rightarrow 168.1$ & 24 & 120 \\
\hline 182 & Imazalil (enilconazole) & $\begin{array}{l}\text { F, MB, } \\
\text { WP, V }\end{array}$ & Approved & Yes & LC & 6.53 & 0.40 & Positive & $297.1 \rightarrow 159.0$ & 20 & $297.1 \rightarrow 69.1$ & 18 & 100 \\
\hline 183 & Imidacloprid & I & Approved & Yes & LC & 3.93 & 0.80 & Positive & $256.0 \rightarrow 175.0$ & 12 & $256.0 \rightarrow 209.0$ & 12 & 110 \\
\hline 184 & Indeno $[1,2,3-c d]$ pyrene & POP & - & No & GC & 19.08 & 0.40 & Positive & $276.0 \rightarrow 274.0$ & 50 & $276.0 \rightarrow 272.0$ & 60 & 70 \\
\hline 185 & Indoxacarb & I & Approved & Yes & LC & 9.49 & 0.20 & Positive & $528.1 \rightarrow 293.1$ & 10 & $528.1 \rightarrow 202.8$ & 48 & 140 \\
\hline 186 & Iprodione & $\begin{array}{l}\mathrm{F}, \mathrm{MB} \text {, } \\
\text { WP }\end{array}$ & $\begin{array}{l}\text { Not } \\
\text { approved }\end{array}$ & Yes & GC & 13.67 & 4.00 & Positive & $314.0 \rightarrow 56.0$ & 20 & $314.0 \rightarrow 245.0$ & 10 & 70 \\
\hline 187 & Iprovalicarb & $\mathrm{F}$ & Approved & Yes & LC & 8.18 & 0.20 & Positive & $321.2 \rightarrow 119.0$ & 15 & $321.2 \rightarrow 202.9$ & 20 & 110 \\
\hline 188 & Isocarbophos & I & $\begin{array}{l}\text { Not } \\
\text { approved }\end{array}$ & Yes & GC & 10.04 & 1.60 & Positive & $230.0 \rightarrow 155.0$ & 25 & $230.0 \rightarrow 198.0$ & 10 & 70 \\
\hline 189 & Isofenphos methyl & I & $\begin{array}{l}\text { Not } \\
\text { approved }\end{array}$ & No & GC & 10.38 & 0.40 & Positive & $199.0 \rightarrow 121.0$ & 10 & $241.0 \rightarrow 121.0$ & 25 & 70 \\
\hline 190 & Isoprothiolane & $\begin{array}{l}\text { F, MB, } \\
\text { WP }\end{array}$ & $\begin{array}{l}\text { Not } \\
\text { approved }\end{array}$ & Yes & LC & 7.94 & 0.10 & Positive & $291.1 \rightarrow 189.0$ & 30 & $291.1 \rightarrow 145.0$ & 36 & 100 \\
\hline 191 & Ivermectin B1a & $\mathrm{V}, \mathrm{AH}, \mathrm{A}$ & Approved & - & LC & 11.52 & 1.60 & Positive & $897.5 \rightarrow 753.5$ & 50 & $897.5 \rightarrow 329.3$ & 60 & 160 \\
\hline 192 & Josamycin & $\mathrm{V}, \mathrm{MB}$ & $\begin{array}{l}\text { Not } \\
\text { approved }\end{array}$ & - & $\mathrm{LC}$ & 7.40 & 0.40 & Positive & $860.5 \rightarrow 173.9$ & 40 & $860.5 \rightarrow 108.9$ & 40 & 200 \\
\hline 193 & Ketoprofen & $\mathrm{V}$, NSAID & Approved & - & LC & 7.34 & 0.40 & Positive & $255.1 \rightarrow 209.1$ & 8 & $255.1 \rightarrow 77.1$ & 48 & 123 \\
\hline 194 & Kresoxim methyl & $\mathrm{F}$ & Approved & Yes & GC & 11.78 & 1.20 & Positive & $116.0 \rightarrow 89.0$ & 15 & $206.0 \rightarrow 131.0$ & 10 & 70 \\
\hline 195 & Leptophos & I & $\begin{array}{l}\text { Not } \\
\text { approved }\end{array}$ & No & GC & 14.58 & 0.80 & Positive & $171.0 \rightarrow 77.1$ & 15 & $377.0 \rightarrow 362.0$ & 20 & 70 \\
\hline
\end{tabular}




\begin{tabular}{|c|c|c|c|c|c|c|c|c|c|c|c|c|c|}
\hline \multirow[t]{2}{*}{ No. } & \multirow[t]{2}{*}{ Compound } & \multirow[t]{2}{*}{ Category $^{\mathrm{a}}$} & \multirow{2}{*}{$\begin{array}{l}\text { Legal } \\
\text { status } \\
\text { in the } \mathrm{EU}^{\mathrm{b}}\end{array}$} & \multirow{2}{*}{$\begin{array}{l}\text { Subjected } \\
\text { to } \mathrm{MRL}^{\mathrm{C}}\end{array}$} & \multirow[t]{2}{*}{ Technique $^{\mathrm{d}}$} & \multirow[t]{2}{*}{ Retention time (min) } & \multirow{2}{*}{$\begin{array}{l}\text { LOQ } \\
(\mathrm{ng} / \mathrm{ml})\end{array}$} & \multirow[t]{2}{*}{ Polarity } & \multicolumn{2}{|l|}{ Quantification } & \multicolumn{2}{|l|}{ Confirmation } & \multirow{2}{*}{$\begin{array}{l}\text { Fragmentor } \\
\text { voltage }(\mathrm{V})\end{array}$} \\
\hline & & & & & & & & & $\begin{array}{l}\text { MRM transition } \\
(m / z)\end{array}$ & $\begin{array}{l}\text { Collision } \\
\text { energy } \\
(\mathrm{eV})\end{array}$ & $\begin{array}{l}\text { MRM } \\
\text { transition } \\
(m / z)\end{array}$ & $\begin{array}{l}\text { Collision } \\
\text { energy } \\
(\mathrm{eV})\end{array}$ & \\
\hline 196 & Levamisole & $\mathrm{V}, \mathrm{AH}$ & Approved & - & LC & 3.12 & 0.20 & Positive & $205.1 \rightarrow 178.1$ & 20 & $205.1 \rightarrow 123.0$ & 32 & 141 \\
\hline 197 & Lincomycin & $\mathrm{V}, \mathrm{MB}$ & Approved & - & LC & 3.50 & 0.40 & Positive & $407.2 \rightarrow 126.1$ & 24 & $407.2 \rightarrow 359.2$ & 16 & 150 \\
\hline 198 & Linuron & $\mathrm{F}$ & Approved & Yes & LC & 7.54 & 0.20 & Positive & $249.0 \rightarrow 160.1$ & 20 & $249.0 \rightarrow 182.3$ & 8 & 120 \\
\hline 199 & Lufenuron & I & $\begin{array}{l}\text { Not } \\
\text { approved }\end{array}$ & Yes & LC & 10.05 & 0.40 & Negative & $509.0 \rightarrow 339.0$ & 5 & $509.0 \rightarrow 326.1$ & 15 & 90 \\
\hline 200 & Malaoxon & I & $\begin{array}{l}\text { Not } \\
\text { approved }\end{array}$ & No & LC & 6.03 & 0.10 & Positive & $315.1 \rightarrow 127.2$ & 12 & $315.1 \rightarrow 99.1$ & 36 & 120 \\
\hline 201 & Malathion & I & $\begin{array}{l}\text { Not } \\
\text { approved }\end{array}$ & Yes & LC & 7.93 & 0.20 & Positive & $348.0 \rightarrow 126.7$ & 15 & $348.0 \rightarrow 285.0$ & 8 & 100 \\
\hline 202 & Mandipropamid & $\mathrm{F}$ & Approved & Yes & LC & 7.90 & 0.10 & Positive & $412.1 \rightarrow 328.1$ & 8 & $412.1 \rightarrow 356.1$ & 4 & 130 \\
\hline 203 & Marbofloxacin & $\mathrm{V}, \mathrm{MB}$ & Approved & - & LC & 3.53 & 2.00 & Positive & $363.2 \rightarrow 72.1$ & 25 & $363.2 \rightarrow 320.1$ & 15 & 134 \\
\hline 204 & Mebendazole & $\mathrm{V}, \mathrm{AH}$ & Approved & - & LC & 6.68 & 0.10 & Positive & $296.1 \rightarrow 264.1$ & 20 & $296.1 \rightarrow 77.0$ & 48 & 151 \\
\hline 205 & Mefenamic acid & V, NSAID & $\begin{array}{l}\text { Not } \\
\text { approved }\end{array}$ & - & LC & 9.52 & 0.40 & Positive & $242.1 \rightarrow 209.1$ & 28 & $242.1 \rightarrow 180.1$ & 0 & 108 \\
\hline 206 & Mefenoxam (metalaxyl-M) & $\mathrm{F}$ & Approved & Yes & LC & 6.95 & 0.10 & Positive & $280.0 \rightarrow 220.0$ & 10 & $280.0 \rightarrow 192.0$ & 15 & 110 \\
\hline 207 & Meloxicam & V, NSAID & Approved & - & LC & 7.17 & 0.20 & Positive & $352.5 \rightarrow 114.8$ & 20 & $352.5 \rightarrow 140.8$ & 20 & 130 \\
\hline 208 & Mepanipyrim & $\begin{array}{l}\text { F, MB, } \\
\text { WP }\end{array}$ & Approved & Yes & GC & 11.13 & 0.40 & Positive & $222.0 \rightarrow 221.0$ & 15 & $222.0 \rightarrow 207.0$ & 15 & 70 \\
\hline 209 & Mepiquat & $\mathrm{H}$ & Approved & Yes & LC & 0.64 & 0.40 & Positive & $114.0 \rightarrow 98.0$ & 36 & $114.0 \rightarrow 70.0$ & 45 & 100 \\
\hline 210 & Metaflumizone & I & Approved & No & LC & 9.94 & 0.20 & Negative & $505.0 \rightarrow 302.0$ & 14 & $541.0 \rightarrow 302.0$ & 20 & 90 \\
\hline 211 & Metaldehyde & $\mathrm{M}$ & Approved & No & LC & 3.87 & 4.00 & Positive & $194.1 \rightarrow 61.9$ & 5 & $194.1 \rightarrow 106.0$ & 5 & 50 \\
\hline 212 & Metconazole & $\mathrm{F}$ & Approved & No & LC & 9.17 & 0.10 & Positive & $320.1 \rightarrow 70.2$ & 33 & $322.1 \rightarrow 70.2$ & 24 & 250 \\
\hline 213 & Methamidophos (two isomers) & $\mathrm{I}, \mathrm{A}$ & $\begin{array}{l}\text { Not } \\
\text { approved }\end{array}$ & Yes & LC & 1.18 & 1.20 & Positive & $142.0 \rightarrow 94.0$ & 12 & $142.0 \rightarrow 125.0$ & 12 & 85 \\
\hline 214 & Methidathion & I, A & $\begin{array}{l}\text { Not } \\
\text { approved }\end{array}$ & Yes & LC & 7.12 & 0.10 & Positive & $320.1 \rightarrow 144.8$ & 8 & $320.1 \rightarrow 85.0$ & 30 & 84 \\
\hline 215 & Methiocarb & $\mathrm{I}, \mathrm{A}, \mathrm{M}$ & $\begin{array}{l}\text { Not } \\
\text { approved }\end{array}$ & Yes & LC & 7.67 & 0.10 & Positive & $226.1 \rightarrow 169.0$ & 4 & $226.1 \rightarrow 121.1$ & 12 & 90 \\
\hline 216 & Methiocarb-sulfoxide & Met & - & Yes & LC & 4.03 & 0.80 & Positive & $242.0 \rightarrow 185.0$ & 22 & $242.0 \rightarrow 122.0$ & 28 & 90 \\
\hline 217 & Methomyl & $\mathrm{I}, \mathrm{A}, \mathrm{AH}$ & $\begin{array}{l}\text { Nor } \\
\text { approved }\end{array}$ & Yes & LC & 3.23 & 0.40 & Positive & $163.1 \rightarrow 88.0$ & 5 & $163.0 \rightarrow 106.0$ & 8 & 80 \\
\hline 218 & Methomyl oxime & Met & - & Yes & LC & 3.25 & 8.00 & Positive & $106.2 \rightarrow 58.1$ & 10 & $106.2 \rightarrow 31.2$ & 20 & 70 \\
\hline 219 & Methoxyfenozide & I & Approved & Yes & LC & 8.00 & 0.10 & Positive & $369.2 \rightarrow 149.0$ & 10 & $369.2 \rightarrow 313.1$ & 15 & 85 \\
\hline 220 & Metoxychlor & POP & - & No & GC & 13.98 & 0.80 & Positive & $227.0 \rightarrow 141.0$ & 20 & $227.0 \rightarrow 169.0$ & 15 & 70 \\
\hline 221 & Metrafenone & $\mathrm{F}$ & Approved & Yes & LC & 9.27 & 0.10 & Positive & $409.1 \rightarrow 209.1$ & 8 & $411.1 \rightarrow 209.1$ & 12 & 108 \\
\hline 222 & Metronidazole & $\mathrm{V}, \mathrm{MB}$ & Approved & - & LC & 2.63 & 0.80 & Positive & $172.1 \rightarrow 128.0$ & 12 & $172.1 \rightarrow 82.1$ & 24 & 98 \\
\hline 223 & Mevinphos (phosdrin) & I, A & $\begin{array}{l}\text { Not } \\
\text { approved }\end{array}$ & No & LC & 4.38 & 0.80 & Positive & $225.0 \rightarrow 193.1$ & 15 & $225.0 \rightarrow 127.0$ & 12 & 65 \\
\hline 224 & Mirex & POP & - & No & GC & 5.66 & 2.00 & Positive & $237.0 \rightarrow 143.0$ & 30 & $274.0 \rightarrow 237.0$ & 10 & 70 \\
\hline 225 & Monocrotophos & I & $\begin{array}{l}\text { Not } \\
\text { approved }\end{array}$ & Yes & LC & 3.31 & 0.80 & Positive & $224.1 \rightarrow 126.8$ & 12 & $224.1 \rightarrow 98.1$ & 15 & 100 \\
\hline 226 & Myclobutanil & $\begin{array}{l}\mathrm{F}, \mathrm{MB} \\
\mathrm{WP}\end{array}$ & Approved & Yes & LC & 8.10 & 0.10 & Positive & $289.1 \rightarrow 70.1$ & 16 & $289.1 \rightarrow 125.1$ & 32 & 110 \\
\hline 227 & $\begin{array}{l}\mathrm{N} \text {-(2,4-dimethylphenyl)- } \mathrm{N}^{\prime} \text {-methylformamidine (DMPF, } \\
\text { metabolite of amitraz) }\end{array}$ & $\mathrm{Met}^{\mathrm{g}}$ & - & No & LC & 3.35 & 0.80 & Positive & $163.1 \rightarrow 122.1$ & 15 & $163.1 \rightarrow 107.1$ & 15 & 100 \\
\hline 228 & $\mathrm{~N}, \mathrm{~N}$-dimethylformamidine (DMF, metabolite of amitraz) & Met $^{\mathrm{g}}$ & - & No & LC & 5.45 & 1.20 & Positive & $150.1 \rightarrow 77.0$ & 40 & $150.1 \rightarrow 105.8$ & 30 & 100 \\
\hline 229 & $\begin{array}{l}\mathrm{N}, \mathrm{N} \text {-dimethyl- } \mathrm{N}^{\prime} \text {-p-tolylsulphamide (DMST, metabolite of } \\
\text { tolyfluanid) }\end{array}$ & $\operatorname{Met}^{\mathrm{i}}$ & - & No & LC & 6.06 & 0.20 & Positive & $215.1 \rightarrow 106.1$ & 10 & $215.1 \rightarrow 151.1$ & 4 & 90 \\
\hline 230 & Nafcillin & $\mathrm{V}, \mathrm{MB}$ & $\begin{array}{l}\text { Not } \\
\text { approved }\end{array}$ & - & LC & 7.33 & 0.80 & Positive & $415.0 \rightarrow 199.1$ & 8 & $415.0 \rightarrow 171.0$ & 36 & 103 \\
\hline 231 & Naphtalene & POP & - & No & GC & 4.45 & 0.80 & Positive & $128.0 \rightarrow 127.0$ & 15 & $128.0 \rightarrow 102.0$ & 25 & 70 \\
\hline 232 & Naproxen & V, NSAID & $\begin{array}{l}\text { Not } \\
\text { approved }\end{array}$ & - & LC & 7.59 & 1.60 & Positive & $231.0 \rightarrow 185.0$ & 10 & $231.1 \rightarrow 169.9$ & 21 & 120 \\
\hline
\end{tabular}




\begin{tabular}{|c|c|c|c|c|c|c|c|c|c|c|c|c|c|}
\hline 233 & Nitenpyram & I & $\begin{array}{l}\text { Not } \\
\text { approved }\end{array}$ & No & LC & 3.30 & 2.00 & Positive & $271.1 \rightarrow 56.1$ & 36 & $271.1 \rightarrow 224.9$ & 12 & 100 \\
\hline 234 & Novobiocin & $\mathrm{V}, \mathrm{MB}$ & $\begin{array}{l}\text { Not } \\
\text { approved }\end{array}$ & - & LC & 9.69 & 0.80 & Positive & $613.2 \rightarrow 218.1$ & 10 & $613.2 \rightarrow 396.1$ & 10 & 150 \\
\hline 235 & Nuarimol & $\begin{array}{l}\text { F, MB, } \\
\text { WP }\end{array}$ & Approved & No & GC & 13.27 & 0.20 & Positive & $235.0 \rightarrow 139.0$ & 15 & $235.0 \rightarrow 111.0$ & 40 & 70 \\
\hline 236 & Ofurace & $\begin{array}{l}\text { F, MB, } \\
\text { WP }\end{array}$ & Approved & No & LC & 5.97 & 0.10 & Positive & $282.0 \rightarrow 159.9$ & 20 & $282.0 \rightarrow 147.9$ & 30 & 100 \\
\hline 237 & Omethoate & I, A & $\begin{array}{l}\text { Not } \\
\text { approved }\end{array}$ & Yes & LC & 2.80 & 0.40 & Positive & $214.1 \rightarrow 124.8$ & 22 & $214.1 \rightarrow 183.0$ & 5 & 100 \\
\hline 238 & Oxadixyl & $\begin{array}{l}\text { F, MB, } \\
\text { WP }\end{array}$ & $\begin{array}{l}\text { Not } \\
\text { approved }\end{array}$ & Yes & LC & 5.43 & 0.20 & Positive & $279.1 \rightarrow 219.2$ & 5 & $279.1 \rightarrow 132.2$ & 32 & 110 \\
\hline 239 & Oxamyl & $\mathrm{I}, \mathrm{A}, \mathrm{AH}$ & Approved & Yes & LC & 2.87 & 0.40 & Positive & $237.1 \rightarrow 72.0$ & 12 & $237.1 \rightarrow 90.0$ & 5 & 70 \\
\hline 240 & Oxfendazole & $\mathrm{V}, \mathrm{AH}$ & Approved & - & LC & 5.61 & 0.10 & Positive & $316.1 \rightarrow 159.0$ & 32 & $316.1 \rightarrow 191.1$ & 16 & 166 \\
\hline 241 & Oxolinic acid & $\mathrm{V}, \mathrm{MB}$ & $\begin{array}{l}\text { Not } \\
\text { approved }\end{array}$ & - & LC & 5.04 & 0.20 & Positive & $262.1 \rightarrow 216.0$ & 32 & $262.1 \rightarrow 160.0$ & 36 & 110 \\
\hline 242 & Oxydemeton methyl & I & $\begin{array}{l}\text { Not } \\
\text { approved }\end{array}$ & Yes & LC & 3.01 & 0.40 & Positive & $247.0 \rightarrow 169.0$ & 12 & $247.0 \rightarrow 109.0$ & 24 & 100 \\
\hline 243 & Oxyfluorfen & $\mathrm{H}$ & Approved & No & GC & 11.68 & 0.40 & Positive & $252.0 \rightarrow 146.0$ & 40 & $300.0 \rightarrow 223.0$ & 15 & 70 \\
\hline 244 & Paclobutrazol & $\mathrm{H}$ & Approved & Yes & LC & 7.89 & 0.40 & Positive & $294.1 \rightarrow 70.1$ & 16 & $294.1 \rightarrow 125.2$ & 36 & 115 \\
\hline 245 & Paraoxon methyl & I & $\begin{array}{l}\text { Not } \\
\text { approved }\end{array}$ & No & GC & 9.00 & 1.60 & Positive & $230.0 \rightarrow 106.0$ & 20 & $230.0 \rightarrow 136.0$ & 5 & 70 \\
\hline 246 & Parathion ethyl & I & $\begin{array}{l}\text { Not } \\
\text { approved }\end{array}$ & No & GC & 9.95 & 1.20 & Positive & $290.9 \rightarrow 109.0$ & 10 & $138.9 \rightarrow 109.0$ & 5 & 70 \\
\hline 247 & Parathion methyl & I & $\begin{array}{l}\text { Not } \\
\text { approved }\end{array}$ & Yes & GC & 9.12 & 0.80 & Positive & $263.0 \rightarrow 109.0$ & 15 & $263.0 \rightarrow 79.0$ & 30 & 70 \\
\hline 248 & PCB 28 & POP & - & Yes & GC & 9.01 & 0.10 & Positive & $256.0 \rightarrow 186.0$ & 25 & $256.0 \rightarrow 151.0$ & 50 & 70 \\
\hline 249 & PCB 52 & POP & - & Yes & GC & 9.58 & 0.20 & Positive & $292.0 \rightarrow 222.0$ & 25 & $292.0 \rightarrow 220.0$ & 25 & 70 \\
\hline 250 & РCB 77 & РОР & - & Yes & GC & 11.73 & 0.20 & Positive & $292.0 \rightarrow 220.0$ & 25 & $292.0 \rightarrow 222.0$ & 25 & 70 \\
\hline 251 & РCВ 81 & РОР & - & Yes & GC & 11.56 & 0.10 & Positive & $292.0 \rightarrow 220.0$ & 25 & $292.0 \rightarrow 222.0$ & 25 & 70 \\
\hline 252 & РCB 101 & POP & - & Yes & GC & 11.08 & 0.20 & Positive & $326.0 \rightarrow 256.0$ & 30 & $328.0 \rightarrow 256.0$ & 30 & 70 \\
\hline 253 & РСB 105 & POP & - & Yes & GC & 12.66 & 0.10 & Positive & $326.0 \rightarrow 256.0$ & 30 & $328.0 \rightarrow 256.0$ & 30 & 70 \\
\hline 254 & РCB 114 & POP & - & Yes & GC & 12.38 & 0.20 & Positive & $326.0 \rightarrow 256.0$ & 30 & $328.0 \rightarrow 256.0$ & 30 & 70 \\
\hline 255 & РCB 118 & РОР & - & Yes & GC & 12.18 & 0.20 & Positive & $326.0 \rightarrow 256.0$ & 30 & $328.0 \rightarrow 256.0$ & 30 & 70 \\
\hline 256 & РCB 123 & POP & - & Yes & GC & 12.10 & 0.40 & Positive & $326.0 \rightarrow 256.0$ & 30 & $328.0 \rightarrow 256.0$ & 30 & 70 \\
\hline 257 & PCB 126 & РОР & - & Yes & GC & 13.23 & 0.20 & Positive & $326.0 \rightarrow 256.0$ & 30 & $328.0 \rightarrow 256.0$ & 30 & 70 \\
\hline 258 & PCB 138 & РОР & - & Yes & GC & 13.07 & 0.10 & Positive & $360.0 \rightarrow 290.0$ & 25 & $360.0 \rightarrow 288.0$ & 25 & 70 \\
\hline 259 & РCB 153 & POP & - & Yes & GC & 12.57 & 0.10 & Positive & $360.0 \rightarrow 290.0$ & 25 & $360.0 \rightarrow 288.0$ & 25 & 70 \\
\hline 260 & РCB 156 & POP & - & Yes & GC & 13.96 & 0.20 & Positive & $360.0 \rightarrow 290.0$ & 25 & $360.0 \rightarrow 288.0$ & 25 & 70 \\
\hline 261 & PCB 157 & POP & - & Yes & GC & 14.07 & 0.40 & Positive & $360.0 \rightarrow 290.0$ & 25 & $360.0 \rightarrow 288.0$ & 25 & 70 \\
\hline 262 & РCB 167 & РОР & - & Yes & GC & 13.55 & 0.10 & Positive & $360.0 \rightarrow 290.0$ & 25 & $360.0 \rightarrow 288.0$ & 25 & 70 \\
\hline 263 & РCB 169 & РОР & - & Yes & GC & 14.61 & 0.20 & Positive & $360.0 \rightarrow 290.0$ & 25 & $360.0 \rightarrow 288.0$ & 25 & 70 \\
\hline 264 & PCB 180 & РОР & - & Yes & GC & 14.25 & 0.10 & Positive & $394.0 \rightarrow 324.0$ & 30 & $394.0 \rightarrow 322.0$ & 30 & 70 \\
\hline 265 & РCB 189 & POP & - & Yes & GC & 15.25 & 0.10 & Positive & $394.0 \rightarrow 324.0$ & 30 & $394.0 \rightarrow 322.0$ & 30 & 70 \\
\hline 266 & Penconazole & $\begin{array}{l}\text { F, MB, } \\
\text { WP }\end{array}$ & Approved & Yes & GC & 10.52 & 0.40 & Positive & $248.0 \rightarrow 157.0$ & 30 & $248.0 \rightarrow 192.0$ & 15 & 70 \\
\hline 267 & Pencycuron & $\begin{array}{l}\text { F, MB, } \\
\text { WP }\end{array}$ & Approved & Yes & LC & 9.33 & 0.10 & Positive & $329.1 \rightarrow 125.1$ & 24 & $329.1 \rightarrow 217.9$ & 12 & 160 \\
\hline 268 & Pendimethalin & $\mathrm{H}$ & Approved & Yes & GC & 10.49 & 0.80 & Positive & $252.0 \rightarrow 162.0$ & 10 & $252.0 \rightarrow 191.0$ & 5 & 70 \\
\hline 269 & Penicillin G & $\mathrm{V}, \mathrm{MB}$ & $\begin{array}{l}\text { Not } \\
\text { approved }\end{array}$ & - & LC & 5.82 & 2.00 & Positive & $335.1 \rightarrow 176.0$ & 10 & $335.1 \rightarrow 160.0$ & 4 & 110 \\
\hline 270 & Penicillin V & $\mathrm{V}, \mathrm{MB}$ & $\begin{array}{l}\text { Not } \\
\text { approved }\end{array}$ & - & LC & 6.47 & 2.00 & Positive & $383.2 \rightarrow 159.9$ & 10 & $383.2 \rightarrow 113.9$ & 40 & 130 \\
\hline 271 & Permethrin & I, A & $\begin{array}{l}\text { Not } \\
\text { approved }\end{array}$ & Yes & GC & 15.69 & 1.20 & Positive & $183.0 \rightarrow 128.0$ & 15 & $183.1 \rightarrow 153.1$ & 15 & 70 \\
\hline 272 & Phenanthrene & POP & - & No & GC & 8.40 & 0.20 & Positive & $178.0 \rightarrow 176.0$ & 35 & $178.0 \rightarrow 152.0$ & 28 & 70 \\
\hline 273 & Phenylbutazone & V, NSAID & Approved & - & LC & 8.25 & 1.60 & Positive & $309.2 \rightarrow 160.2$ & 20 & $309.2 \rightarrow 77.1$ & 55 & 140 \\
\hline 274 & Phosalone & I, A & Not & No & LC & 9.20 & 0.20 & Positive & $385.1 \rightarrow 182.0$ & 20 & $385.1 \rightarrow 110.9$ & 55 & 80 \\
\hline
\end{tabular}




\begin{tabular}{|c|c|c|c|c|c|c|c|c|c|c|c|c|c|}
\hline \multirow[t]{2}{*}{ No. } & \multirow[t]{2}{*}{ Compound } & \multirow[t]{2}{*}{ Category $^{a}$} & \multirow{2}{*}{$\begin{array}{l}\text { Legal } \\
\text { status } \\
\text { in the } \mathrm{EU}^{\mathrm{b}}\end{array}$} & \multirow{2}{*}{$\begin{array}{l}\text { Subjected } \\
\text { to } \mathrm{MRL}^{\mathrm{c}}\end{array}$} & \multirow[t]{2}{*}{ Technique $^{\mathrm{d}}$} & \multirow[t]{2}{*}{ Retention time (min) } & \multirow{2}{*}{$\begin{array}{l}\text { LOQ } \\
(\mathrm{ng} / \mathrm{ml})\end{array}$} & \multirow[t]{2}{*}{ Polarity } & \multicolumn{2}{|l|}{ Quantification } & \multicolumn{2}{|l|}{ Confirmation } & \multirow{2}{*}{$\begin{array}{l}\text { Fragmentor } \\
\text { voltage (V) }\end{array}$} \\
\hline & & & & & & & & & $\begin{array}{l}\text { MRM transition } \\
(m / z)\end{array}$ & $\begin{array}{l}\text { Collision } \\
\text { energy } \\
(\mathrm{eV})\end{array}$ & $\begin{array}{l}\text { MRM } \\
\text { transition } \\
(m / z)\end{array}$ & $\begin{array}{l}\text { Collision } \\
\text { energy } \\
(\mathrm{eV})\end{array}$ & \\
\hline 275 & Phosmet & I, A & Approved & Yes & LC & 7.34 & 0.20 & Positive & $318.0 \rightarrow 159.9$ & 16 & $318.0 \rightarrow 133.0$ & 40 & 90 \\
\hline 276 & Phosmet oxon & Met & - & Yes & LC & 5.36 & 0.20 & Positive & $302.0 \rightarrow 160.0$ & 10 & $302.0 \rightarrow 133.0$ & 38 & 60 \\
\hline 277 & Piperacillin & $\mathrm{V}, \mathrm{MB}$ & $\begin{array}{l}\text { Not } \\
\text { approved }\end{array}$ & - & LC & 5.68 & 0.40 & Positive & $518.2 \rightarrow 143.0$ & 16 & $518.2 \rightarrow 160.0$ & 4 & 121 \\
\hline 278 & Pirimicarb & I & Approved & Yes & LC & 5.11 & 0.10 & Positive & $239.1 \rightarrow 72.1$ & 20 & $239.1 \rightarrow 182.1$ & 12 & 100 \\
\hline 279 & Pirimiphos ethyl & $\mathrm{I}, \mathrm{A}$ & $\begin{array}{l}\text { Not } \\
\text { approved }\end{array}$ & No & GC & 10.26 & 0.10 & Positive & $318.0 \rightarrow 166.0$ & 15 & $318.0 \rightarrow 182.0$ & 15 & 70 \\
\hline 280 & Pirimiphos methyl & I, A & Approved & Yes & LC & 9.13 & 0.10 & Positive & $306.1 \rightarrow 164.0$ & 20 & $306.1 \rightarrow 108.1$ & 32 & 100 \\
\hline 281 & Prochloraz & $\begin{array}{l}\text { F, MB, } \\
\text { WP }\end{array}$ & Approved & No & $\mathrm{LC}$ & 9.08 & 0.10 & Positive & $376.0 \rightarrow 308.0$ & 10 & $376.0 \rightarrow 70.1$ & 20 & 100 \\
\hline 282 & Procymidone & $\begin{array}{l}\text { F, MB, } \\
\text { WP }\end{array}$ & $\begin{array}{l}\text { Not } \\
\text { approved }\end{array}$ & Yes & GC & 10.80 & 1.60 & Positive & $283.0 \rightarrow 67.0$ & 40 & $283.0 \rightarrow 68.0$ & 25 & 70 \\
\hline 283 & Profenofos & $\mathrm{I}, \mathrm{A}$ & $\begin{array}{l}\text { Not } \\
\text { approved }\end{array}$ & Yes & LC & 9.75 & 0.10 & Positive & $375.0 \rightarrow 305.0$ & 20 & $373.0 \rightarrow 303.0$ & 20 & 100 \\
\hline 284 & Propamocarb & $\mathrm{F}$ & Approved & Yes & LC & 2.85 & 0.40 & Positive & $189.2 \rightarrow 102.0$ & 12 & $189.2 \rightarrow 144.0$ & 8 & 110 \\
\hline 285 & Propargite & A & $\begin{array}{l}\text { Not } \\
\text { approved }\end{array}$ & Yes & LC & 10.37 & 0.10 & Positive & $368.2 \rightarrow 231.1$ & 4 & $368.2 \rightarrow 175.0$ & 12 & 88 \\
\hline 286 & Propiconazole & A & $\begin{array}{l}\text { Not } \\
\text { approved }\end{array}$ & Yes & LC & 9.01 & 0.40 & Positive & $342.0 \rightarrow 69.0$ & 21 & $342.0 \rightarrow 159.0$ & 39 & 90 \\
\hline 287 & Propoxur & I & $\begin{array}{l}\text { Not } \\
\text { approved }\end{array}$ & No & LC & 5.83 & 0.10 & Positive & $210.1 \rightarrow 168.1$ & 35 & $210.1 \rightarrow 65.1$ & 40 & 70 \\
\hline 288 & Propyzamide (pronamide) & $\mathrm{H}$ & Approved & Yes & LC & 7.92 & 0.10 & Positive & $256.1 \rightarrow 190.0$ & 16 & $256.1 \rightarrow 173.0$ & 25 & 90 \\
\hline 289 & Proquinazid & $\mathrm{F}$ & Approved & Yes & GC & 13.32 & 0.20 & Positive & $288.0 \rightarrow 245.0$ & 15 & $288.0 \rightarrow 217.0$ & 30 & 70 \\
\hline 290 & Prothioconazol & $\mathrm{F}$ & Approved & Yes & GC & 11.85 & 0.40 & Positive & $186.0 \rightarrow 49.0$ & 20 & $186.0 \rightarrow 53.0$ & 25 & 70 \\
\hline 291 & Prothiophos & $\mathrm{F}$ & $\begin{array}{l}\text { Not } \\
\text { approved }\end{array}$ & No & GC & 11.45 & 0.40 & Positive & $266.9 \rightarrow 221.0$ & 35 & $162.0 \rightarrow 63.1$ & 30 & 70 \\
\hline 292 & Pymetrozine & I & $\begin{array}{l}\text { Not } \\
\text { approved }\end{array}$ & Yes & LC & 2.74 & 0.80 & Positive & $218.1 \rightarrow 105.0$ & 20 & $218.1 \rightarrow 78.0$ & 52 & 120 \\
\hline 293 & Pyraclostrobin & $\mathrm{F}$ & Approved & Yes & LC & 9.15 & 0.10 & Positive & $388.1 \rightarrow 193.8$ & 8 & $388.1 \rightarrow 163.1$ & 28 & 120 \\
\hline 294 & Pyrazophos & $\begin{array}{l}\text { F, MB, } \\
\text { WP }\end{array}$ & $\begin{array}{l}\text { Not } \\
\text { approved }\end{array}$ & No & LC & 9.22 & 0.10 & Positive & $374.1 \rightarrow 222.1$ & 23 & $374.1 \rightarrow 194.0$ & 32 & 100 \\
\hline 295 & Pyrene & POP & - & No & GC & 11.13 & 0.20 & Positive & $202.0 \rightarrow 201.0$ & 27 & $202.0 \rightarrow 200.0$ & 45 & 70 \\
\hline 296 & Pyridaben & $\mathrm{I}, \mathrm{A}$ & Approved & Yes & LC & 10.75 & 0.10 & Positive & $365.2 \rightarrow 309.0$ & 8 & $309.1 \rightarrow 147.0$ & 16 & 168 \\
\hline 297 & Pyridaphenthion & $\mathrm{I}, \mathrm{A}$ & $\begin{array}{l}\text { Not } \\
\text { approved }\end{array}$ & No & $\mathrm{LC}$ & 8.11 & 0.20 & Positive & $341.0 \rightarrow 189.0$ & 22 & $341.0 \rightarrow 205.0$ & 34 & 100 \\
\hline 298 & Pyrimethanil & $\mathrm{F}$ & Approved & Yes & GC & 8.27 & 0.20 & Positive & $198.0 \rightarrow 118.0$ & 40 & $198.0 \rightarrow 158.0$ & 20 & 70 \\
\hline 299 & Pyriproxifen & I & Approved & Yes & LC & 10.07 & 0.10 & Positive & $322.2 \rightarrow 96.0$ & 12 & $322.2 \rightarrow 184.9$ & 24 & 80 \\
\hline 300 & Quinalfos & $\mathrm{I}, \mathrm{A}$ & $\begin{array}{l}\text { Not } \\
\text { approved }\end{array}$ & No & LC & 8.72 & 0.20 & Positive & $299.1 \rightarrow 96.9$ & 30 & $299.1 \rightarrow 147.1$ & 20 & 130 \\
\hline 301 & Quinoxyfen & $\mathrm{F}$ & $\begin{array}{l}\text { Not } \\
\text { approved }\end{array}$ & Yes & LC & 10.13 & 0.10 & Positive & $308.0 \rightarrow 197.0$ & 32 & $308.2 \rightarrow 161.8$ & 55 & 120 \\
\hline 302 & Rifampicin & $\mathrm{V}, \mathrm{MB}$ & $\begin{array}{l}\text { Not } \\
\text { approved }\end{array}$ & - & LC & 7.89 & 0.80 & Positive & $823.5 \rightarrow 791.4$ & 15 & $823.5 \rightarrow 399.1$ & 25 & 160 \\
\hline 303 & Rotenone & $\mathrm{I}, \mathrm{R}$ & $\begin{array}{l}\text { Not } \\
\text { approved }\end{array}$ & No & LC & 8.64 & 0.40 & Positive & $395.1 \rightarrow 213.1$ & 20 & $395.1 \rightarrow 192.1$ & 25 & 150 \\
\hline 304 & Roxithromycin & $\mathrm{V}, \mathrm{MB}$ & $\begin{array}{l}\text { Not } \\
\text { approved }\end{array}$ & - & LC & 7.67 & 0.80 & Positive & $838.5 \rightarrow 158.1$ & 40 & $838.5 \rightarrow 116.1$ & 55 & 200 \\
\hline 305 & Sarafloxacin & $\mathrm{V}, \mathrm{MB}$ & $\begin{array}{l}\text { Not } \\
\text { approved }\end{array}$ & - & LC & 4.16 & 4.00 & Positive & $386.1 \rightarrow 342.1$ & 16 & $386.1 \rightarrow 299.1$ & 28 & 144 \\
\hline 306 & Simazine & I & $\begin{array}{l}\text { Not } \\
\text { approved }\end{array}$ & No & LC & 5.81 & 0.20 & Positive & $202.4 \rightarrow 68.1$ & 30 & $202.4 \rightarrow 68.1$ & 20 & 120 \\
\hline 307 & Spinosad (two isomers) & $\mathrm{I}, \mathrm{V}$ & Approved & Yes & LC & $9.10 / 9.43$ & 0.10 & Positive & $732.4 \rightarrow 142.0$ & 22 & $732.4 \rightarrow 98.0$ & 60 & 130 \\
\hline 308 & Spiramycin (two isomers) & $\mathrm{V}, \mathrm{MB}$ & Approved & - & LC & $4.58 / 4.90$ & 0.40 & Positive & $439.1 \rightarrow 101.1$ & 20 & $439.1 \rightarrow 88.0$ & 50 & 70 \\
\hline
\end{tabular}




\begin{tabular}{|c|c|c|c|c|c|c|c|c|c|c|c|c|c|}
\hline 309 & Spirodiclofen & A & Approved & Yes & LC & 10.50 & 0.80 & Positive & $411.1 \rightarrow 71.2$ & 15 & $411.1 \rightarrow 313.0$ & 5 & 110 \\
\hline 310 & Spiromesifen & I & Approved & Yes & LC & 10.27 & 0.20 & Positive & $388.0 \rightarrow 273.0$ & 25 & $273.0 \rightarrow 187.0$ & 15 & 110 \\
\hline 311 & Spiroxamine & $\mathrm{F}$ & Approved & Yes & LC & 7.55 & 0.10 & Positive & $298.3 \rightarrow 144.1$ & 16 & $298.3 \rightarrow 100.1$ & 32 & 120 \\
\hline 312 & Strychnine & $\mathrm{R}$ & $\begin{array}{l}\text { Not } \\
\text { approved }\end{array}$ & No & LC & $3.00 / 3.61$ & 0.80 & Positive & $335.1 \rightarrow 184.0$ & 45 & $335.1 \rightarrow 156.0$ & 40 & 105 \\
\hline 313 & Sulfacetamide & $\mathrm{V}, \mathrm{MB}$ & $\begin{array}{l}\text { Not } \\
\text { approved }\end{array}$ & - & LC & 2.13 & 0.40 & Positive & $215.3 \rightarrow 155.9$ & 10 & $215.3 \rightarrow 92.0$ & 20 & 90 \\
\hline 314 & Sulfachloropiridacine & $\mathrm{V}, \mathrm{MB}$ & $\begin{array}{l}\text { Not } \\
\text { approved }\end{array}$ & - & LC & 3.77 & 0.80 & Positive & $285.0 \rightarrow 156.0$ & 12 & $285.0 \rightarrow 92.1$ & 28 & 101 \\
\hline 315 & Sulfadiacine & $\mathrm{V}, \mathrm{MB}$ & Approved & - & LC & 2.80 & 0.80 & Positive & $251.0 \rightarrow 92.0$ & 28 & $251.0 \rightarrow 156.0$ & 12 & 111 \\
\hline 316 & Sulfadimetoxine & $\mathrm{V}, \mathrm{MB}$ & Approved & - & LC & 4.81 & 0.10 & Positive & $311.0 \rightarrow 92.0$ & 32 & $311.0 \rightarrow 156.0$ & 16 & 139 \\
\hline 317 & Sulfadoxine & $\mathrm{V}, \mathrm{MB}$ & Approved & - & LC & 4.12 & 0.10 & Positive & $311.1 \rightarrow 92.0$ & 32 & $311.1 \rightarrow 156.0$ & 16 & 126 \\
\hline 318 & Sulfameracine & $\mathrm{V}, \mathrm{MB}$ & $\begin{array}{l}\text { Not } \\
\text { approved }\end{array}$ & - & LC & 3.26 & 0.20 & Positive & $265.0 \rightarrow 92.0$ & 28 & $265.0 \rightarrow 156.0$ & 12 & 126 \\
\hline 319 & Sulfametacine & $\mathrm{V}, \mathrm{MB}$ & $\begin{array}{l}\text { Not } \\
\text { approved }\end{array}$ & - & LC & 3.44 & 0.20 & Positive & $279.1 \rightarrow 186.0$ & 12 & $279.1 \rightarrow 92.0$ & 32 & 134 \\
\hline 320 & Sulfametizole & $\mathrm{V}, \mathrm{MB}$ & $\begin{array}{l}\text { Not } \\
\text { approved }\end{array}$ & - & LC & 3.37 & 0.80 & Positive & $271.0 \rightarrow 92.0$ & 28 & $271.0 \rightarrow 155.9$ & 8 & 103 \\
\hline 321 & Sulfametoxazole & $\mathrm{V}, \mathrm{MB}$ & Approved & - & LC & 3.93 & 0.40 & Positive & $254.0 \rightarrow 92.0$ & 28 & $254.0 \rightarrow 156.0$ & 12 & 111 \\
\hline 322 & Sulfametoxipiridacine & $\mathrm{V}, \mathrm{MB}$ & $\begin{array}{l}\text { Not } \\
\text { approved }\end{array}$ & - & LC & 3.45 & 0.40 & Positive & $281.0 \rightarrow 155.9$ & 12 & $281.0 \rightarrow 92.1$ & 28 & 121 \\
\hline 323 & Sulfamonomethoxine & $\mathrm{V}, \mathrm{MB}$ & $\begin{array}{l}\text { Not } \\
\text { approved }\end{array}$ & - & LC & 4.11 & 1.20 & Positive & $281.1 \rightarrow 156.0$ & 14 & $281.1 \rightarrow 92.1$ & 32 & 120 \\
\hline 324 & Sulfapyridine & $\mathrm{V}, \mathrm{MB}$ & $\begin{array}{l}\text { Not } \\
\text { approved }\end{array}$ & - & LC & 2.82 & 0.40 & Positive & $250.0 \rightarrow 156.0$ & 12 & $250.0 \rightarrow 92.0$ & 28 & 126 \\
\hline 325 & Sulfaquinoxaline & $\mathrm{V}, \mathrm{MB}$ & Approved & - & LC & 4.99 & 0.40 & Positive & $301.0 \rightarrow 156.0$ & 12 & $301.0 \rightarrow 92.1$ & 32 & 159 \\
\hline 326 & Sulfatiazole & $\mathrm{V}, \mathrm{MB}$ & $\begin{array}{l}\text { Not } \\
\text { approved }\end{array}$ & - & $\mathrm{LC}$ & 2.98 & 0.40 & Positive & $256.0 \rightarrow 92.0$ & 28 & $256.0 \rightarrow 156.0$ & 12 & 106 \\
\hline 327 & Sulfisoxazole & $\mathrm{V}, \mathrm{MB}$ & $\begin{array}{l}\text { Not } \\
\text { approved }\end{array}$ & - & LC & 4.12 & 0.80 & Positive & $268.0 \rightarrow 156.0$ & 8 & $268.0 \rightarrow 92.1$ & 24 & 106 \\
\hline 328 & Tebuconazole & $\mathrm{I}, \mathrm{A}$ & Approved & Yes & LC & 8.92 & 0.80 & Positive & $308.2 \rightarrow 70.2$ & 22 & $308.2 \rightarrow 125.1$ & 53 & 120 \\
\hline 329 & Tebufenocide & I & Approved & Yes & LC & 8.66 & 0.10 & Positive & $353.1 \rightarrow 132.9$ & 22 & $353.1 \rightarrow 297.1$ & 20 & 90 \\
\hline 330 & Tebufenpyrad & A & Approved & Yes & LC & 9.88 & 0.10 & Positive & $334.2 \rightarrow 117.0$ & 47 & $334.2 \rightarrow 145.0$ & 37 & 180 \\
\hline 331 & Teflubenzuron & I & $\begin{array}{l}\text { Not } \\
\text { approved }\end{array}$ & Yes & $\mathrm{LC}$ & 10.01 & 1.20 & Negative & $379.0 \rightarrow 339.0$ & 15 & $379.0 \rightarrow 196.0$ & 25 & 100 \\
\hline 332 & Tefluthrin & I & Approved & Yes & GC & 8.42 & 0.10 & Positive & $177.0 \rightarrow 127.0$ & 15 & $177.0 \rightarrow 87.0$ & 15 & 70 \\
\hline 333 & Telodrin (isobenzan) & I & $\begin{array}{l}\text { Not } \\
\text { approved }\end{array}$ & No & GC & 10.14 & 0.80 & Positive & $310.8 \rightarrow 240.8$ & 25 & $310.8 \rightarrow 274.8$ & 5 & 70 \\
\hline 334 & Terbufos & $\mathrm{I}, \mathrm{AH}$ & $\begin{array}{l}\text { Not } \\
\text { approved }\end{array}$ & No & GC & 8.15 & 0.20 & Positive & $231.0 \rightarrow 97.0$ & 20 & $231.0 \rightarrow 129.0$ & 15 & 70 \\
\hline 335 & Terbuthylazine & $\mathrm{H}$ & Approved & Yes & GC & 8.12 & 0.40 & Positive & $214.0 \rightarrow 104.0$ & 20 & $214.0 \rightarrow 132.0$ & 10 & 70 \\
\hline 336 & Tetrachlorvinphos & I & $\begin{array}{l}\text { Not } \\
\text { approved }\end{array}$ & No & LC & 8.72 & 0.40 & Positive & $367.0 \rightarrow 127.0$ & 16 & $365.0 \rightarrow 127.0$ & 16 & 110 \\
\hline 337 & Tetraconazole & $\mathrm{F}, \mathrm{H}$ & Approved & Yes & GC & 10.04 & 0.20 & Positive & $336.0 \rightarrow 204.0$ & 35 & $336.0 \rightarrow 218.0$ & 20 & 70 \\
\hline 338 & Tetradifon & A & $\begin{array}{l}\text { Not } \\
\text { approved }\end{array}$ & No & GC & 14.36 & 0.40 & Positive & $158.9 \rightarrow 111.0$ & 20 & $354.0 \rightarrow 159.0$ & 10 & 70 \\
\hline 339 & Tetramethrin & I & $\begin{array}{l}\text { Not } \\
\text { approved }\end{array}$ & No & GC & 13.87 & 1.60 & Positive & $164.0 \rightarrow 77.0$ & 30 & $164.0 \rightarrow 107.0$ & 15 & 70 \\
\hline 340 & Thiabendazole & $\mathrm{AH}, \mathrm{V}$ & Approved & Yes & LC & 3.50 & 0.20 & Positive & $202.0 \rightarrow 175.0$ & 24 & $202.0 \rightarrow 131.0$ & 36 & 170 \\
\hline 341 & Thiacloprid & I & Approved & No & LC & 4.80 & 0.20 & Positive & $253.0 \rightarrow 126.0$ & 16 & $253.0 \rightarrow 90.0$ & 40 & 140 \\
\hline 342 & Thiamethoxam & I & $\begin{array}{l}\text { Not } \\
\text { approved }\end{array}$ & Yes & LC & 3.59 & 0.80 & Positive & $292.0 \rightarrow 211.1$ & 8 & $292.0 \rightarrow 132.0$ & 22 & 80 \\
\hline 343 & Thiophanate methyl & I & Approved & Yes & LC & 5.87 & 0.20 & Positive & $343.0 \rightarrow 151.0$ & 20 & $343.0 \rightarrow 93.0$ & 46 & 90 \\
\hline 344 & Tolclofos methyl & $\begin{array}{l}\text { F, MB, } \\
\text { WP }\end{array}$ & Approved & Yes & GC & 9.21 & 0.10 & Positive & $265.0 \rightarrow 93.0$ & 30 & $265.0 \rightarrow 220.0$ & 25 & 70 \\
\hline 345 & Tolfenamic acid & V, NSAID & $\begin{array}{l}\text { Not } \\
\text { approved }\end{array}$ & - & LC & 9.80 & 0.40 & Negative & $260.0 \rightarrow 216.1$ & 8 & $260.0 \rightarrow 35.1$ & 20 & 108 \\
\hline 346 & Triadimefon & $\begin{array}{l}\text { F, MB, } \\
\text { WP }\end{array}$ & $\begin{array}{l}\text { Not } \\
\text { approved }\end{array}$ & Yes & LC & 8.03 & 0.40 & Positive & $294.1 \rightarrow 69.3$ & 20 & $294.1 \rightarrow 197.2$ & 15 & 100 \\
\hline
\end{tabular}




\begin{tabular}{|c|c|c|c|c|c|c|c|c|c|c|c|c|c|}
\hline \multirow[t]{2}{*}{ No. } & \multirow[t]{2}{*}{ Compound } & \multirow[t]{2}{*}{ Category $^{a}$} & \multirow{2}{*}{$\begin{array}{l}\text { Legal } \\
\text { status } \\
\text { in the } \mathrm{EU}^{\mathrm{b}}\end{array}$} & \multirow{2}{*}{$\begin{array}{l}\text { Subjected } \\
\text { to } \mathrm{MRL}^{\mathrm{c}}\end{array}$} & \multirow[t]{2}{*}{ Technique $^{d}$} & \multirow[t]{2}{*}{ Retention time (min) } & \multirow{2}{*}{$\begin{array}{l}\text { LOQ } \\
(\mathrm{ng} / \mathrm{ml})\end{array}$} & \multirow[t]{2}{*}{ Polarity } & \multicolumn{2}{|l|}{ Quantification } & \multicolumn{2}{|l|}{ Confirmation } & \multirow{2}{*}{$\begin{array}{l}\text { Fragmentor } \\
\text { voltage (V) }\end{array}$} \\
\hline & & & & & & & & & $\begin{array}{l}\text { MRM transition } \\
(m / z)\end{array}$ & $\begin{array}{l}\text { Collision } \\
\text { energy } \\
(\mathrm{eV})\end{array}$ & $\begin{array}{l}\text { MRM } \\
\text { transition } \\
(m / z)\end{array}$ & $\begin{array}{l}\text { Collision } \\
\text { energy } \\
(\mathrm{eV})\end{array}$ & \\
\hline 347 & Triadimenol & $\begin{array}{l}\text { F, MB, } \\
\text { WP }\end{array}$ & $\begin{array}{l}\text { Not } \\
\text { approved }\end{array}$ & Yes & LC & 8.22 & 0.40 & Positive & $296.1 \rightarrow 70.0$ & 10 & $298.1 \rightarrow 70.0$ & 10 & 80 \\
\hline 348 & Triazophos (hostathion) & $\mathrm{I}, \mathrm{A}$ & $\begin{array}{l}\text { Not } \\
\text { approved }\end{array}$ & Yes & LC & 8.18 & 0.10 & Positive & $314.1 \rightarrow 162.0$ & 19 & $314.1 \rightarrow 118.9$ & 35 & 100 \\
\hline 349 & Trichlorfon & $\mathrm{I}, \mathrm{AH}, \mathrm{V}$ & $\begin{array}{l}\text { Not } \\
\text { approved }\end{array}$ & No & LC & 4.06 & 1.20 & Positive & $256.9 \rightarrow 109.0$ & 12 & $258.9 \rightarrow 109.0$ & 12 & 170 \\
\hline 350 & Trifloxystrobin & $\mathrm{F}$ & Approved & Yes & LC & 9.50 & 0.10 & Positive & $409.1 \rightarrow 186.0$ & 12 & $409.1 \rightarrow 145.0$ & 52 & 110 \\
\hline 351 & Triflumizole & $\mathrm{F}$ & Approved & No & LC & 9.53 & 0.10 & Positive & $346.1 \rightarrow 278.0$ & 4 & $345.9 \rightarrow 73.0$ & 15 & 80 \\
\hline 352 & Triflumuron & I & Approved & Yes & LC & 9.19 & 0.40 & Positive & $359.0 \rightarrow 156.0$ & 8 & $359.0 \rightarrow 139.0$ & 32 & 120 \\
\hline 353 & Trifluralin & $\mathrm{H}$ & $\begin{array}{l}\text { Not } \\
\text { approved }\end{array}$ & No & GC & 7.27 & 0.20 & Positive & $264.0 \rightarrow 160.0$ & 15 & $306.0 \rightarrow 264.0$ & 5 & 70 \\
\hline 354 & Trimethoprim & $\mathrm{V}, \mathrm{MB}$ & Approved & - & LC & 3.45 & 0.80 & Positive & $291.2 \rightarrow 123.0$ & 24 & $291.2 \rightarrow 230.1$ & 20 & 162 \\
\hline 355 & Triticonazole & $\mathrm{F}$ & Approved & No & LC & 8.38 & 0.40 & Positive & $318.1 \rightarrow 70.1$ & 33 & $320.1 \rightarrow 70.1$ & 16 & 110 \\
\hline 356 & Tylmicosin & $\mathrm{V}, \mathrm{MB}$ & Approved & - & LC & 5.52 & 1.60 & Positive & $869.6 \rightarrow 174.1$ & 48 & $869.6 \rightarrow 696.4$ & 44 & 294 \\
\hline 357 & Tylosin & $\mathrm{V}, \mathrm{MB}$ & Approved & - & LC & 6.76 & 0.80 & Positive & $916.5 \rightarrow 174.1$ & 40 & $916.5 \rightarrow 772.4$ & 28 & 210 \\
\hline 358 & Vinclozolin & $\begin{array}{l}\text { F, MB, } \\
\text { WP }\end{array}$ & $\begin{array}{l}\text { Not } \\
\text { approved }\end{array}$ & Yes & GC & 9.10 & 0.20 & Positive & $212.0 \rightarrow 145.0$ & 25 & $212.0 \rightarrow 109.0$ & 50 & 70 \\
\hline 359 & Warfarin & $\mathrm{R}$ & $\begin{array}{l}\text { Not } \\
\text { approved }\end{array}$ & No & LC & 7.86 & 0.10 & Negative & $307.1 \rightarrow 161.1$ & 20 & $307.1 \rightarrow 250.1$ & 20 & 140 \\
\hline \multirow[t]{14}{*}{360} & Zoxamide & $\mathrm{F}$ & Approved & No & LC & 9.03 & 0.40 & Positive & $336.0 \rightarrow 187.1$ & 25 & $187.1 \rightarrow 88.9$ & 40 & 200 \\
\hline & Acenaphthene-d10 & P-IS & - & - & GC & 6.16 & - & Positive & $164.0 \rightarrow 162.0$ & 18 & $164.0 \rightarrow 160.0$ & 35 & 70 \\
\hline & Atrazine-d5 & P-IS & - & - & GC & 7.95 & - & Positive & $205.1 \rightarrow 127.1$ & 14 & $205.1 \rightarrow 105.0$ & 14 & 70 \\
\hline & Atrazine-d5 & P-IS & - & - & LC & 6.74 & - & Positive & $221.2 \rightarrow 179.0$ & 15 & $221.2 \rightarrow 69.1$ & 50 & 90 \\
\hline & Carbendazim-d3 & P-IS & - & - & LC & 2.91 & - & Positive & $195.1 \rightarrow 160.1$ & 15 & $195.1 \rightarrow 131.9$ & 30 & 100 \\
\hline & Chorpyrifos-d10 & P-IS & - & - & GC & 9.94 & - & Positive & $324.0 \rightarrow 260.0$ & 35 & $324.0 \rightarrow 195.0$ & 55 & 70 \\
\hline & Chrysene-d12 & P-IS & - & - & GC & 13.86 & - & Positive & $240.0 \rightarrow 238.0$ & 20 & $240.0 \rightarrow 236.0$ & 38 & 70 \\
\hline & Cyromazine-d4 & P-IS & - & - & LC & 1.24 & - & Positive & $171.0 \rightarrow 129.0$ & 15 & $171.0 \rightarrow 86.0$ & 15 & 100 \\
\hline & Diazinon-d10 & P-IS & - & - & GC & 8.29 & - & Positive & $314.0 \rightarrow 199.0$ & 5 & $314.0 \rightarrow 183.0$ & 15 & 70 \\
\hline & Diazinon-d10 & P-IS & - & - & LC & 8.93 & - & Positive & $315.2 \rightarrow 170.1$ & 20 & $315.2 \rightarrow 154.3$ & 20 & 100 \\
\hline & Linuron-d3 & P-IS & - & - & LC & 7.54 & - & Positive & $255.1 \rightarrow 185.0$ & 15 & $255.1 \rightarrow 159.8$ & 15 & 100 \\
\hline & PCB 200 & P-IS & - & - & GC & 14.51 & - & Positive & $429.8 \rightarrow 359.8$ & 30 & $427.8 \rightarrow 357.8$ & 30 & 70 \\
\hline & Phenanthrene-d10 & P-IS & - & - & GC & 8.40 & - & Positive & $188.0 \rightarrow 186.0$ & 20 & $188.0 \rightarrow 184.0$ & 35 & 70 \\
\hline & Pirimicarb-d6 & P-IS & - & - & LC & 5.12 & - & Positive & $245.2 \rightarrow 185.0$ & 5 & $245.2 \rightarrow 78.2$ & 30 & 70 \\
\hline
\end{tabular}

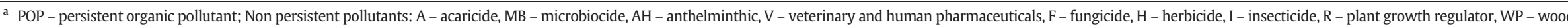
preservative, PHP - post-harvest preservative, M - Molluscicide, Met - metabolite, NSAID - nonsteroidal anti-inflammatory drug, GC - glucocorticoid, P-IS - procedural internal standard.

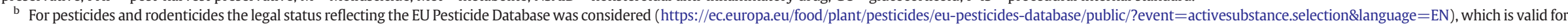
the entire EU. For veterinary drugs, the marketing status in Spain is specified, as shown in the Cima vet search engine of the Spanish agency for drugs and health products (https://cimavet.aemps.es/cimavet/publico/home.html).

c Pesticide considered in the coordinated multi-annual plan of the EU for the investigation of residues in food of vegetable or animal origin during the years 2020 , 2021 and 2022 (Regulation CE/2019/533).

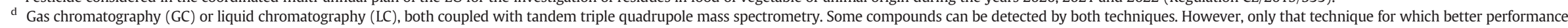
(lower LOQ best recovery or lower RSD) has been indicated.

${ }^{\mathrm{e}}$ Isomer beta (beta-cyfluthrin) is approved until 31 October 2020

f Isomer beta (beta-cypermethrin) has switch to the "not approved" status since September 2017.

$\mathrm{g}$ The exposure to the acaricide amitraz is evaluated through the presence of these two major metabolites.

The exposure to dichlofluanide is evaluated through the presence of this metabolite.

${ }^{i}$ The exposure to tolyfluanide is evaluated through the presence of this metabolite. 
polarity range, while matrix co-eluents extracts the least (Sell et al., 2018) being at the same time suitable for LC and GC chromatography. Both methods have been developed for the analysis of pesticides in foods of plant origin, using $10 \mathrm{~g}$ of sample, and involve a clean-up step with secondary primary amine (PSA) for the removal of organic acids, fatty acids, sugars; and C18 for the elimination of lipids and sterols; and/or graphitized carbon (GC) for pigment removal. However, our previous experience demonstrated that these adsorbents retain various polar compounds (for example, a large number of pharmaceuticals and some POPs) that are of interest to this method. Therefore, we decided not to test clean-up with these adsorbents. However, a novel sorbent, the EMR-Lipid, was recently launched for the clean-up of fatty sample extracts, such as whole blood (Agilent, 2015). Therefore, a clean-up step with EMR-lipid was tested at two levels (2 y $20 \mathrm{ng} / \mathrm{ml}$ ) in triplicate. As different authors have indicated that, when using the EN method, the extraction efficiency of acetonitrile improves in the acidic condition, this method was tested in the presence or absence of $1 \%$ FA (EC, 2015). Ten and one grams of whole blood were tested in these initial experiments ( 3 replicates at two concentrations $(2-20 \mathrm{ng} / \mathrm{ml})$, analyzed in duplicate). We did not find differences between them. Therefore, we continued next experiments with $1 \mathrm{~g}$ of whole blood instead of $10 \mathrm{~g}$.

We made the decision on which method to choose based on two criteria. First, we chose the method that extracted more compounds above $60 \%$ recovery (all quantifications were performed against matrix-matched calibrators). The second criterion was based on the ion abundance. According to these criteria, the clean-up with EMRlipid was ruled out since 97 and 113 compounds were lost or poorly recovered with the AOAC 2007.01 method and the UNE-EN 15662: 2019 method, respectively. One-step application of QuEChERS gave excellent results with both protocols, with an ability to adequately extract a similar number of compounds ( 360 with the AOAC method vs. 354 with the EN method). However, ion abundance was better for the majority of compounds with the AOAC method in the presence of $1 \% \mathrm{FA}$, so this was the chosen method. In a further step, the possibility of refining the method by modifying the percentage of FA was tested $(0.0,0.2$, $0.5,1,2.5$ and $5 \%$ ), but no better results than those of $1 \%$ FA were found.

One of the most important objectives of this methodological development was to minimize the amount of sample employed. Therefore, several additional experiments were carried out, in which the amount of sample was progressively decreased $(1,0.5,0.25,0.1)$. Obviously, the amount of salts was proportionally decreased. The minimum amount of sample that did not affect the performance of the extraction method was $0.25 \mathrm{~g}$ of whole blood, so all validation of the method was performed using these conditions.

\subsection{Validation}

This method allows the simultaneous quantification of 360 chemicals, including 56 POPs, 205 agricultural pesticides, 11 rodenticides, 67 pharmaceuticals, and 21 metabolites. The detailed list of compounds, together with the specific category of use, and the technique of instrumental analysis, are presented in Table 1. Besides, for agricultural pesticides, the legal status in the EU and whether or not it is included in the coordinated multi-annual plan of the EU for the investigation of residues in food, is also indicated in Table 1. Data obtained during the validation process meet both, the criteria of the SANTE 2017 guidance document and those of the Scientific Working Group for Forensic Toxicology (EC, 2019b; SWGTOX, 2013).

Identity was evaluated through ion qualifier ratio ( \pm 30 average ion qualifier ratio of matrix-matched standards from the same sequence), retention time deviation ( $\pm 0.1 \mathrm{~min}$ ), peak shape, and signal/noise ratio ( $\mathrm{s} / \mathrm{n}>3$ for all ions, peak-to-peak algorithm). At least two transitions per compound were optimized. Transition with higher response and less noise at the lowest calibration point was used as a quantifier and at least one more as a qualifier (confirmation). Whenever possible, the quasimolecular ion was used in the identification of the compounds. The quantification and confirmation transitions that were selected for each compound are shown in Table 1. On the other hand, selectivity, which is the recommended term in analytical chemistry to express the extent of interferences (EC, 2019b), was evaluated by assessing the absence or presence of interfering or co-eluting chromatographic peaks at the retention time of the target analytes in the blank samples extracted by the optimized micro QuEChERS method.

Linearity was assessed within the range of concentrations that were considered appropriate for the purpose of biomonitoring ( 0.1 to $20 \mathrm{ng} / \mathrm{ml}$ ). Within this range, 12 matrix-matched calibration points in quintuplicate were evaluated. All compounds showed acceptable linearity, with the lowest correlation coefficient $\left(R^{2}\right)$ values being those of sarafloxacin, naphthalene, benzo[k]fluoranthene, fluvalinate, and fipronil sulfide ( $R^{2}$ about 0.93 ). It is noteworthy, however, that the values of $R^{2}$ were within the range $0.97-0.99$ for nearly $95 \%$ of compounds $\left(\mathrm{n}=342\right.$ ). Detailed $\mathrm{R}^{2}$ values for all the compounds are shown in Table 1 of the accompanying Data in Brief article.

The influence of the matrix components on the performance of the method was evaluated by applying the extraction method to a sufficient quantity of whole blood to produce a blank matrix extract, which was subsequently fortified at three levels for the mixture of 360 chemicals $(0.2,2$, and $20 \mathrm{ng} / \mathrm{ml})$ and quantified against a calibration curve prepared in the solvent. Matrix effect (ME) was observed both for compounds analyzed by LC-MS/MS and GC-MS/MS. Strong or medium signal suppression was demonstrated for $13.88 \%$ of compounds, and enhancement for $29.44 \%$ of compounds. For 204 pollutants the ME was considered negligible $(-20 \%<\mathrm{ME}<20 \%$ ). However, as for the rest 156 chemical, there was a significant $\mathrm{ME}$, and it was concluded that matrix-matched calibration had to be used to compensate matrix interferences. All the detailed data of ME for individual compounds in whole blood are graphically shown in Fig. 1 of the accompanying article in Data in Brief.

The average recovery and precision (at least 5 fortification levels, each in quintuplicate) obtained were satisfactory for 356 pollutants, as they ranged from 76.6 to $119.5 \%$ with intraday relative standard deviations (RSD) ranging from 0.1 to $19.6 \%$, and interday RSD ranging from 0.08 to $19.2 \%$. Four pollutants did not strictly meet the validation criteria included in the SANTE guide (recoveries in the range $70-120 \%$, and RSD $<20 \%$ ). However, due to its importance in biomonitoring studies, we consider it important to include in the method compounds whose recoveries were lower or higher than those established in the recommendations, but which were highly reproducible (RSD $<15 \%$ ). Thus, marbofloxacin (bias 62\%, RSD 14.5\%), beta hexachlorocyclohexane (bias $132.6 \%$, RSD 14.5\%), spirodiclofen (bias 134.4\%, RSD 8.8\%), and heptachlor (bias 139.4\%, RSD 6.7\%) were also included. All detailed validation data for the five levels of fortification are shown in Table 1 of the accompanying article in Data in Brief. For some compounds with higher LOQs, fewer levels are displayed, as no data is included in the table for levels $<$ LOQ.

In the validation process, the possibility of carryover was also assessed. For this, blank matrix extracts were analyzed immediately after injecting the highest point of the calibration curve (also prepared in the matrix). According to the guidelines, it is acceptable for validation if carryover after the highest calibrator does not exceed $10 \%$ of the signal of the lowest calibrator, and in our case, this condition was met at $20 \mathrm{ng} / \mathrm{ml}$ for all analytes, except for fenbutatin oxide. The carryover effect for this pesticide disappeared completely at the second injection of blank matrix. To assess the need for additional clean-up measures for samples with medium to high levels of contaminants levels, we conducted additional experiments in which the signal from the blank matrix was evaluated after the injection of $100 \mathrm{ng} / \mathrm{ml}$ of the chemical mixture in whole blood. In this case, in addition to fenbutatin oxide, a low carryover was observed for brodifacoum, chlorophacinone, danofloxacin, difloxacin, enrofloxacin, flocoumafen, marbofloxacin, and sarafloxacin. However, the signal disappeared completely after the second injection of blank also for these compounds. 

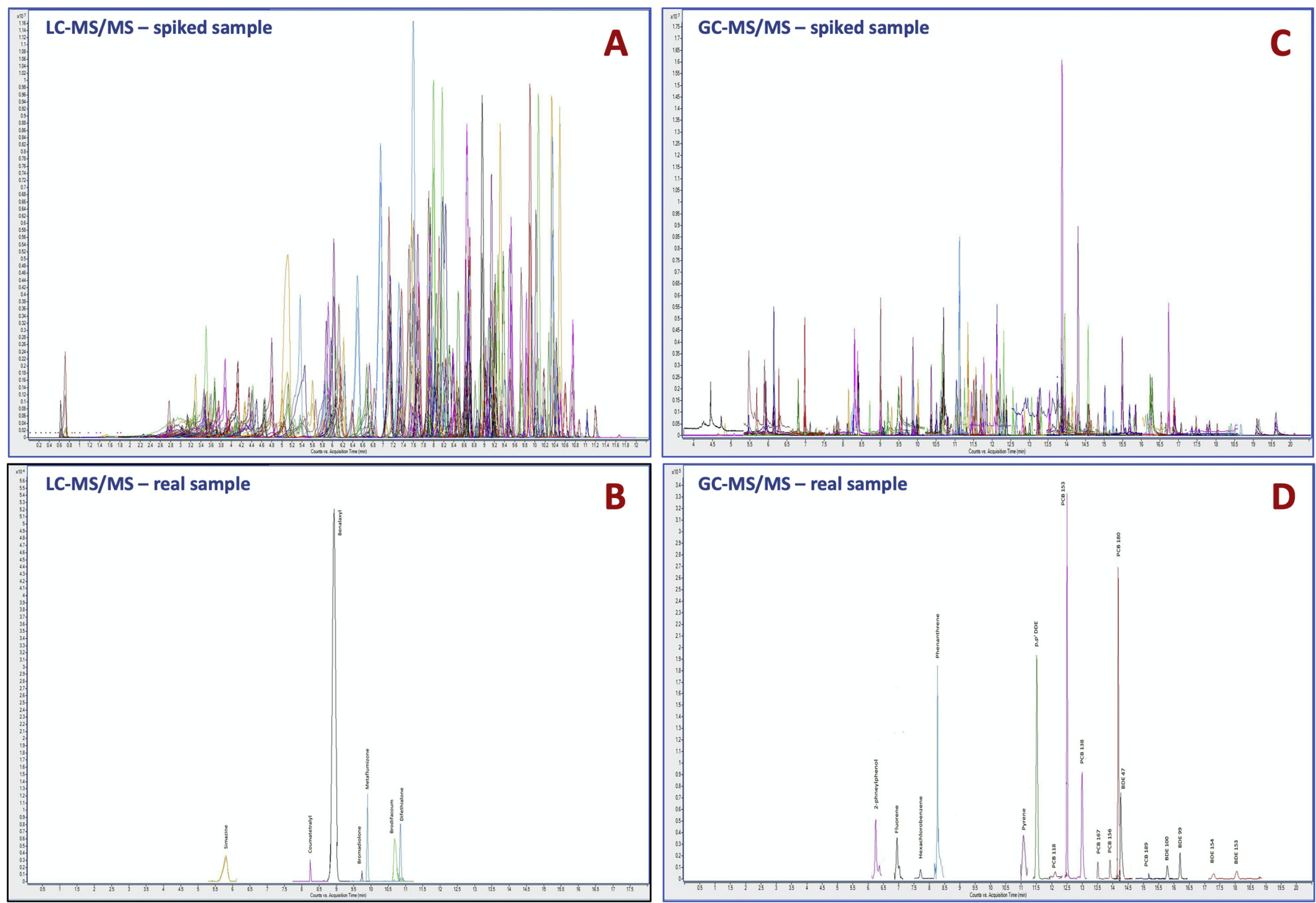

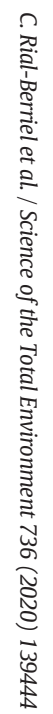

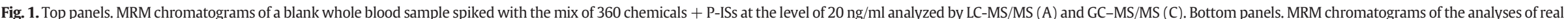
samples (barn owl and common kestrel) by LC-MS/MS (B) and by GC-MS/MS (D). 
The LOQ of this method was calculated over five runs of five fortified blank matrix samples, within the low working range (below $5 \mathrm{ng} / \mathrm{ml}$ ), of three different sources (chicken, goat, and a mixture of both), as recommended (SWGTOX, 2013). The lowest non-zero calibrator approximation was employed to calculate de LOQs. This means that the lowest point of the calibration curve that complied identity, bias and precision criteria was set as the LOQ for a given compound. The expanded method uncertainty (MU) was calculated according to the formula specified in the SANTE guide (1st approach). As this is a new method development, no data of proficiency tests or independent reference materials were available. Therefore, reproducibility RSD was employed, as indicated in the SANTE guide, and an expanded covered factor $\mathrm{k}=2$ was chosen (EC, 2019b). The MU was below 58\% in all cases. As shown in Table 1, the developed method is very sensitive and allows the quantitative analysis at very low levels. Despite the high number of chemicals included, and the small sample volume employed, $95 \%$ compounds can be quantified at LOQs $<1.5 \mathrm{ng} / \mathrm{ml}$. This makes the method very appropriate for the biomonitoring of toxic chemicals in wildlife.

\subsection{Application of the method to a series of blood samples of wild birds}

This method was applied to a serie of 148 samples of whole blood collected in 2018 and 2019 from several species of nocturnal and diurnal raptors belonging to the Group of Rehabilitation of the Native Fauna and its Habitat (GREFA, Majadahonda, Spain). The whole blood samples do not correspond to a homogeneous series of individuals but include birds from 2 different species (Falco tinnunculus and Tyto alba), including both chickens and adults, and both males and females. These data are presented for the sole purpose of demonstrating the potential of the method for biomonitoring contaminants in wildlife. The individual data obtained for each of the individuals are presented in Tables 2-5 of the accompanying article in Data in Brief.

Fig. 1 shows the typical chromatograms of the spiked at $20 \mathrm{ng} / \mathrm{ml}$ whole blood samples obtained by the LC-MS/MS and GC-MS/MS analyses (top panels), and the chromatograms of two different positive samples for 25 pollutants (Tyto alba and Falco tinnunculus, bottom panels), as an example of the application of the method. Fig. 2 shows the data on the number of contaminants detected per sample. In all the samples, at least three of the contaminants were detected, with a maximum of 25 contaminants detected in the barn owl shown in Fig. 1. The median value of the number of pollutants per sample was 7 .

In total, 51 different compounds were detected, which represents $14 \%$ of the chemicals included in the method. Contaminants belonging to 4 of the five groups under study (POPs, agricultural insecticides, rodenticides, and pharmaceuticals) were detected. However, none of the

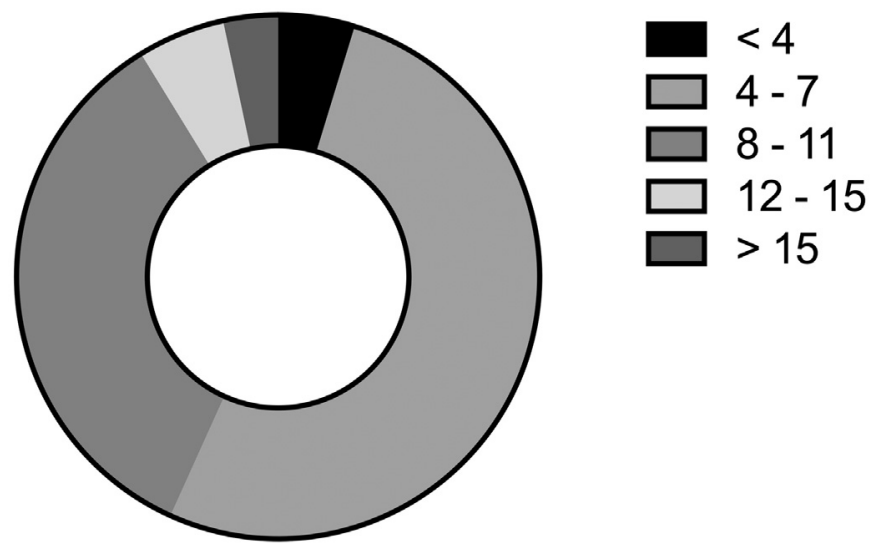

\section{Total $=148$}

Fig. 2. Occurrence of environmental pollutants in the blood of a series of 148 raptors. metabolites (nor the parent compounds) used as exposure biomarkers were detected in any of the samples. Tables 2 and 3 show the results of the contaminants found, along with the mean, median, percentiles, and detection frequency values throughout the series. As expected, of the six compounds that were detected in $>50 \%$ of the samples, five were POPs (phenanthrene, pyrene, fluorene, hexachlorobenzene, and $\mathrm{p}, \mathrm{p}^{\prime}$-DDE) (Table 2). However, it is striking that a contaminant that is neither persistent nor semi-persistent, such as 2-phenylphenol (2PHP), appeared in $96 \%$ of raptor blood samples (Table 3). $2 \mathrm{PHP}$ is a biocide used as preservative and surface disinfectant on fibers and other materials in households, hospitals, and other places, and is recognized as a potential endocrine disruptor (Scientific Committee On Consumer and Bernauer, 2016). Other authors have also reported that 2PHP is a highly prevalent pollutant in biota samples, such as river fish of different species, in which it is found in up to $100 \%$ of the samples (Peng et al., 2018). The rest of the POPs that were detected were the ones that have also been reported most frequently in other series of birds of prey (Espin et al., 2018; Garcia-Heras et al., 2018; Jaspers et al., 2013; Luzardo et al., 2014b; Ortiz-Santaliestra et al., 2015).

Concerning the rest of the agricultural pesticides, the case of benalaxyl is particularly remarkable, since it was detected in $23 \%$ of the individuals analyzed, albeit at low concentrations (Table 3). Benalaxyl is a widely used agricultural fungicide, and both, this agrochemical and, in particular, its metabolites, have been classified as endocrine disruptors endowed with potent anti-estrogenic activity (Ji et al., 2020). However, this pesticide is not routinely included in biomonitoring studies, despite studies on its toxicity to wildlife (Wang et al., 2014). The finding of such a high detection frequency of this fungicide in birds of prey samples indicates that this product has an extensive penetration in the food chains and the ecosystem and also, demonstrates the great utility of the method that we have developed for ecotoxicology studies. On the other hand, diphenylamine was found in $11.5 \%$ of the samples. Its principal use has been as a post-harvest preservative (mostly apples and pears), although its use is no longer authorized in the EU, and therefore such a high frequency of detection in wildlife samples is shocking. The percentage of raptor blood samples that tested positive for this residue is very similar to that of positives in fresh vegetables produced in countries where it is still authorized (Mutengwe et al., 2016). To our knowledge, this is the first study in which the presence of diphenylamine in the blood of raptors is reported. However, the presence of diphenylamine has been reported in gray partridge eggs from agricultural ecosystems (Bro et al., 2015). Moreover, the fact that it has also been found in herbs growing in agricultural areas (Malinowska and Jankowski, 2015), suggests that, independently from its origin, this compound is likely to easily penetrate the food chain (plants, arthropods, rodents, etc...), thus possibly reaching raptors. Metaflumizone was also found in a similar percentage of raptors (10.8\%). Although it is not possible to know the exact origin, it is a permitted insecticide of great use in agriculture, and it has been reported to have a persistence of several days in soil (Chatterjee and Gupta, 2013). Therefore, it also seems possible that this pesticide penetrates the raptors' trophic chain. Five other agricultural pesticides were detected less frequently (Table 3). Among them, it is worth noting simazine, which is another unauthorized pesticide in the EU, and which is of concern because it is a proven endocrine disruptor (Orton et al., 2009). Simazine was detected in $4.73 \%$ of the samples ( $n=7$ individuals).

Although the penetration of rodenticides into the trophic chain is a known fact (Plaza et al., 2019; Ruiz-Suarez et al., 2014; SanchezBarbudo et al., 2012; Seljetun et al., 2019), it is still surprising that residues of at least one of these compounds have been found in almost $15 \%$ of the birds in this series. More than one compound was found in 8 of the individuals (from 2 to 4 compounds). It should be noted that the sample used has been the blood of live animals and not liver samples where these compounds tend to concentrate. These results indicate that these animals are constantly exposed to these rodenticides through feeding, even from the time they are in the nest. In addition, there are 
Table 2

Persistent and semi-persistent organic pollutants detected in 148 blood $\mathrm{s} \rightarrow$ amples of raptors.

\begin{tabular}{|c|c|c|c|c|c|}
\hline \multirow[t]{2}{*}{ Pollutant } & \multirow[t]{2}{*}{ Frequency } & \multicolumn{4}{|c|}{ Concentrations in samples with residues } \\
\hline & & Mean \pm SD & Median & 95th percentile & Max \\
\hline Phenanthrene & 95.95 & $0.75 \pm 0.40$ & 0.61 & 1.46 & 2.25 \\
\hline Pyrene & 95.95 & $0.23 \pm 0.13$ & 0.21 & 0.45 & 0.91 \\
\hline Fluorene & 91.22 & $0.55 \pm 0.27$ & 0.47 & 1.12 & 1.50 \\
\hline Hexachlorobencene & 68.92 & $0.60 \pm 0.72$ & 0.38 & 1.78 & 5.49 \\
\hline Dichlorodiphenyldichloroethylene (p,p' DDE) & 51.89 & $0.52 \pm 0.87$ & 0.20 & 2.90 & 4.40 \\
\hline РСВ 153 & 35.81 & $0.55 \pm 1.01$ & 0.21 & 3.02 & 5.17 \\
\hline PCB 138 & 31.08 & $0.33 \pm 0.56$ & 0.15 & 1.68 & 2.87 \\
\hline PCB 180 & 30.73 & $0.76 \pm 1.64$ & 0.23 & 5.66 & 6.88 \\
\hline Fluoranthene & 19.59 & $0.27 \pm 0.06$ & 0.26 & 0.37 & 0.43 \\
\hline Acenaphtylene & 17.57 & $0.31 \pm 0.08$ & 0.33 & 0.43 & 0.45 \\
\hline РCB 189 & 10.14 & $0.25 \pm 0.17$ & 0.18 & 0.56 & 0.58 \\
\hline PCB 118 & 6.76 & $0.20 \pm 0.16$ & 0.13 & 0.50 & 0.50 \\
\hline BDE 99 & 6.08 & $0.22 \pm 0.20$ & 0.10 & 0.57 & 0.68 \\
\hline Acenaphthene & 5.41 & $0.29 \pm 0.13$ & 0.30 & 0.37 & 0.51 \\
\hline PCB 167 & 5.41 & $0.20 \pm 0.10$ & 0.22 & 0.33 & 0.36 \\
\hline BDE 100 & 4.73 & $0.14 \pm 0.08$ & 0.10 & 0.26 & 0.33 \\
\hline РCB 156 & 4.05 & $0.23 \pm 0.13$ & 0.21 & 0.42 & 0.49 \\
\hline BDE 153 & 3.38 & $0.20 \pm 0.00$ & 0.20 & 0.20 & 0.20 \\
\hline BDE 47 & 2.03 & $0.20 \pm 0.00$ & 0.20 & 0.20 & 0.20 \\
\hline Naphtalene & 2.03 & $1.43 \pm 1.07$ & 0.83 & 2.49 & 2.67 \\
\hline Dichlorodiphenyldichloroethane ( $\mathrm{p}, \mathrm{p}^{\prime}$ DDD) & $0.68^{\mathrm{a}}$ & 0.65 & 0.65 & - & - \\
\hline Hexachlorocyclohexane (alpha) & $0.68^{\mathrm{a}}$ & 0.40 & 0.40 & - & - \\
\hline Hexaclorocyclohexane (beta) & $0.68^{\mathrm{a}}$ & 5.95 & 5.95 & - & - \\
\hline РСВ 28 & $0.68^{\mathrm{a}}$ & 0.10 & 0.10 & - & - \\
\hline PCB 101 & $0.68^{\mathrm{a}}$ & 0.20 & 0.20 & - & - \\
\hline
\end{tabular}

a These compounds were detected in only one individual each.

Table 3

Non persistent pesticides and veterinary drugs detected in 148 blood samples of raptors.

\begin{tabular}{|c|c|c|c|c|c|c|c|c|}
\hline & \multirow[t]{2}{*}{ Category $^{\mathrm{a}}$} & \multirow[t]{2}{*}{ Legal status in the $\mathrm{EU}^{\mathrm{b}}$} & \multirow[t]{2}{*}{ Subjected to MRL ${ }^{\mathrm{c}}$} & \multirow[t]{2}{*}{ Frequency } & \multicolumn{4}{|c|}{ Concentrations in samples with residues } \\
\hline & & & & & Mean \pm SD & Median & 95th percentile & Max \\
\hline \multicolumn{9}{|l|}{ Agricultural pesticides } \\
\hline 2-Phenylphenol & $\mathrm{F}$ & Approved & Yes & 95.95 & $1.02 \pm 1.19$ & 0.59 & 4.19 & 5.88 \\
\hline Benalaxyl & $\mathrm{F}$ & Approved & No & 22.97 & $0.13 \pm 0.04$ & 0.10 & 0.21 & 0.26 \\
\hline Diphenylamine & PHP & Not approved & Yes & 11.49 & $0.37 \pm 0.20$ & 0.31 & 0.63 & 0.82 \\
\hline Metaflumizone & $\mathrm{I}, \mathrm{V}$ & Approved & No & 10.81 & $0.31 \pm 0.21$ & 0.20 & 0.70 & 0.92 \\
\hline Simazine & I & Not approved & No & 4.73 & $0.25 \pm 0.07$ & 0.23 & 0.36 & 0.39 \\
\hline Metrafenone & $\mathrm{F}$ & Approved & Yes & 2.70 & $0.10 \pm 0.01$ & 0.10 & 0.10 & 0.10 \\
\hline Thiacloprid & I & Approved & No & 2.70 & $0.83 \pm 1.17$ & 0.31 & 2.25 & 2.57 \\
\hline Coumaphos & $\mathrm{I}, \mathrm{A}$ & Not approved & No & 1.35 & $0.15 \pm 0.05$ & 0.15 & 0.18 & 0.19 \\
\hline Atrazine & $\mathrm{H}$ & Not approved & No & $0.68^{d}$ & 0.12 & 0.12 & - & - \\
\hline \multicolumn{9}{|l|}{ Rodenticides } \\
\hline Brodifacoum & $\mathrm{R}$ & Not approved & No & 7.43 & $4.38 \pm 9.55$ & 0.80 & 19.55 & 32.73 \\
\hline Difenacoum & $\mathrm{R}$ & Not approved & No & 3.38 & $0.40 \pm 0.01$ & 0.40 & 0.41 & 0.42 \\
\hline Bromadiolone & $\mathrm{R}$ & Approved & No & 2.03 & $0.41 \pm 0.01$ & 0.40 & 0.42 & 0.42 \\
\hline Coumatetralyl & $\mathrm{R}$ & Not approved & No & 2.03 & $41.56 \pm 60.78$ & 13.02 & 101.53 & 111.36 \\
\hline Coumachlor & $\mathrm{R}$ & Not approved & No & $0.68^{d}$ & 5.84 & 5.84 & - & - \\
\hline Difethialone & $\mathrm{R}$ & Not approved & No & $0.68^{d}$ & 1.77 & 1.77 & - & - \\
\hline Flocoumafen & $\mathrm{R}$ & Not approved & No & $0.68^{d}$ & 0.20 & 0.20 & - & - \\
\hline \multicolumn{9}{|l|}{ Pharmaceuticals } \\
\hline Levamisole & $\mathrm{V}$ & Approved & - & 8.11 & $0.29 \pm 0.10$ & 0.27 & 0.34 & 0.52 \\
\hline Fenbendazole & V & Approved & - & 2.70 & $0.10 \pm 0.00$ & 0.10 & 0.10 & 0.10 \\
\hline Enrofloxacin & V & Approved & - & 1.35 & $1.20 \pm 0.00$ & 1.20 & 1.20 & 1.20 \\
\hline Eprinomectin & $\mathrm{V}$ & Approved & - & 1.35 & $0.32 \pm 0.01$ & 0.32 & 0.32 & 0.33 \\
\hline Flumequine & V & Approved & - & 1.35 & $0.10 \pm 0.00$ & 0.10 & 0.10 & 0.10 \\
\hline Sulfadiacine & V & Approved & - & 1.35 & $7.27 \pm 9.28$ & 7.27 & 9.42 & 13.69 \\
\hline Albendazole & V & Approved & - & $0.68^{d}$ & 0.10 & 0.10 & - & - \\
\hline Dexamethasone & V & Approved & - & $0.68^{d}$ & 0.40 & 0.40 & - & - \\
\hline Mebendazole & V & Approved & - & $0.68^{d}$ & 0.25 & 0.25 & - & - \\
\hline Sulfacloropiridacine & V & Not approved & - & $0.68^{d}$ & 0.80 & 0.80 & - & - \\
\hline Sulfapyridine & $\mathrm{V}$ & Not approved & - & $0.68^{d}$ & 0.40 & 0.40 & - & - \\
\hline
\end{tabular}

${ }^{\mathrm{a}} \mathrm{A}$ - acaricide, $\mathrm{B}$ - bactericide, $\mathrm{AH}$ - anthelminthic, $\mathrm{V}$ - veterinary and human pharmaceuticals, $\mathrm{F}$ - fungicide, $\mathrm{H}$ - herbicide, I - insecticide, $\mathrm{R}$ - plant growth regulator, WP - wood preservative, PHP - post-harvest preservative, M - Molluscicide, Met - metabolite.

b For pesticides and rodenticides the legal status reflecting the EU Pesticide Database was considered (https://ec.europa.eu/food/plant/pesticides/eu-pesticides-database/public/?

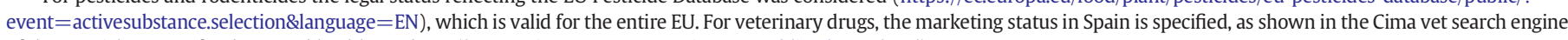
of the Spanish agency for drugs and health products (https://cimavet.aemps.es/cimavet/publico/home.html).

c Pesticide considered in the coordinated multi-annual plan of the EU for the investigation of residues in food of vegetable or animal origin during the years 2020,2021 and 2022 (Regulation CE/2019/533).

d These compounds were detected in only one individual each. 
two other very striking events, such as the fact that six of the seven rodenticides detected in this series are not authorized for agricultural or environmental use in the EU, and also that one of them - brodifacoum - has been the most frequently detected in this series of birds of prey (11 individuals).

Finally, although detection frequencies were low in almost all cases, up to 11 different pharmaceuticals were detected in this series of birds of prey blood samples (Table 3). As in the previous cases, the fact that the sample analyzed is blood implies that the exposure to these contaminants has been recent (and probably regular), possibly only a few hours before sampling. More in-depth studies are needed to assess the toxicological significance of these findings. Still, it is worth noting some data such as the high frequency of detection with which levamisole has appeared in this series (12 individuals with levels $>$ LOQ). To the best of our knowledge, this is the first study that shows data about levamisole in wild raptors. However, some other authors have indicated that this is a prevalent environmental pollutant, and have reported its presence in non-target wild organisms, such as marine mollusks and fish (Moreno-Gonzalez et al., 2016), with frequencies even higher than those reported in this series.

\section{Conclusions}

The current method, with a one-step miniaturized QuEChERS sample preparation, followed by both LC-MS/MS and GC-MS/MS analyses, allows the simultaneous determination of 360 toxic or potentially toxic environmental pollutants in small amounts of whole blood $(250 \mu \mathrm{l})$. The analytical scope of this optimized and fully validated method includes a vast number of chemicals of environmental concern for wildlife, and also for humans. Thus, it includes: i) the most relevant POPs (organochlorine pesticides, polychlorinated biphenyls, polybrominated diphenyl ethers, and polycyclic aromatic hydrocarbons); ii) almost $90 \%$ of the active substances of the plant protection products included in the coordinated multi-annual plan of the EU for the investigation of residues in food of vegetable or animal origin; iii) the most commonly employed chemicals that are involved in deliberate poisoning of wildlife; iv) the most widely used anticoagulant rodenticides; v) pharmaceuticals, including many of those of major use in veterinary practice; vi) and a suite of metabolites that can be used as biomarkers of exposure. The application of the method to actual raptors samples allows to verify its suitability for biomonitoring studies and to glimpse its potential for obtaining valuable exposure data in ecotoxicological studies.

\section{CRediT authorship contribution statement}

Cristian Rial-Berriel: Conceptualization, Investigation, Formal analysis, Writing - original draft, Writing - review \& editing. Andrea AcostaDacal: Investigation, Formal analysis, Writing - original draft, Writing review \& editing. Manuel Zumbado: Investigation, Writing - original draft, Writing - review \& editing. Octavio P. Luzardo: Conceptualization, Investigation, Formal analysis, Writing - original draft, Writing - review \& editing.

\section{Declaration of competing interest}

The authors declare no conflict of interest. This is an independent research. Mention of trade names or commercial products does not constitute endorsement or recommendation for use.

\section{Acknowledgements}

This research was supported by the University of Las Palmas de Gran Canaria via a doctoral grant to the first author Cristian Rial-Berriel (ULPGC-012-2016), and also supported by the Spanish Ministry of Education, Culture and Sports via a doctoral grant to the co-first author
Andrea Acosta-Dacal (FPU16-01888). The authors would like to thank people from Grupo de Rehabilitación de la Fauna Autóctona y su Hábitat (GREFA, Madrid, Spain), specially Dr. Fernando González González, and Mrs. Natalia Pastor Tiburón. We would also like to thank Mrs. Ana Macías Montes and Dr. Luis Alberto Henríquez Hernández for their assistance in the Laboratory of Toxicology of University of Las Palmas de Gran Canaria.

\section{References}

Agilent, 2015. Technical Note - Recommended Protocols for Enhanced Matrix Removal Lipid. Agilent Technologies, Inc, USA (2015).

Allender, W.J., Keegan, J., 1992. Chromatographic analysis of cis- and trans-mevinphos in poisoned wildlife. J. Chromatogr. 609, 315-320.

Anastassiades, M., Lehotay, S.J., Stajnbaher, D., Schenck, F.J., 2003. Fast and easy multiresidue method employing acetonitrile extraction/partitioning and "dispersive solid-phase extraction" for the determination of pesticide residues in produce. J. AOAC Int. 86, 412-431.

Arnold, K.E., Brown, A.R., Ankley, G.T., Sumpter, J.P., 2014. Medicating the environment: assessing risks of pharmaceuticals to wildlife and ecosystems. Philos. Trans. R. Soc. Lond. Ser. B Biol. Sci. 369.

Bartikova, H., Skalova, L., Stuchlikova, L., Vokral, I., Vanek, T., Podlipna, R., 2015. Xenobiotic-metabolizing enzymes in plants and their role in uptake and biotransformation of veterinary drugs in the environment. Drug Metab. Rev. 47, 374-387.

Bille, L., Toson, M., Mulatti, P., Dalla Pozza, M., Capolongo, F., Casarotto, C., Ferre, N., Angeletti, R., Gallocchio, F., Binato, G., 2016. Epidemiology of animal poisoning: an overview on the features and spatio-temporal distribution of the phenomenon in the north-eastern Italian regions. Forensic Sci. Int. 266, 440-448.

Blanco, G., Junza, A., Barron, D., 2017. Occurrence of veterinary pharmaceuticals in golden eagle nestlings: unnoticed scavenging on livestock carcasses and other potential exposure routes. Sci. Total Environ. 586, 355-361.

Bro, E., Millot, F., Decors, A., Devillers, J., 2015. Quantification of potential exposure of gray partridge (Perdix perdix) to pesticide active substances in farmlands. Sci. Total Environ. 521-522, 315-325

Brown, P., Charlton, A., Cuthbert, M., Barnett, L., Ross, L., Green, M., Gillies, L., Shaw, K., Fletcher, M., 1996. Identification of pesticide poisoning in wildlife. J. Chromatogr. A 754, 463-478.

Brown, P.M., Turnbull, G., Charman, S., Charlton, A.J., Jones, A., 2005. Analytical methods used in the United Kingdom Wildlife Incident Investigation Scheme for the detection of animal poisoning by pesticides. J. AOAC Int. 88, 204-220.

Bucchia, M., Camacho, M., Santos, M.R., Boada, L.D., Roncada, P., Mateo, R., OrtizSantaliestra, M.E., Rodriguez-Estival, J., Zumbado, M., Oros, J., Henriquez-Hernandez, L.A., Garcia-Alvarez, N., Luzardo, O.P., 2015. Plasma levels of pollutants are much higher in loggerhead turtle populations from the Adriatic Sea than in those from open waters (Eastern Atlantic Ocean). Sci. Total Environ. 523, 161-169.

Chatterjee, N.S., Gupta, S., 2013. Persistence of metaflumizone on cabbage (Brassica oleracea Linne) and soil, and its risk assessment. Environ. Monit. Assess. 185, 6201-6208.

Cuthbert, R.J., Taggart, M.A., Prakash, V., Chakraborty, S.S., Deori, P., Galligan, T., Kulkarni, M., Ranade, S., Saini, M., Sharma, A.K., Shringarpure, R., Green, R.E., 2014. Avian scavengers and the threat from veterinary pharmaceuticals. Philos. Trans. R. Soc. Lond. Ser. B Biol. Sci. 369

EC, 2015. Analysis of Acidic Pesticides Using QuEChERS (EN15662) and Acidified QuEChERS Method. EU Reference Laboratories for Residues of Pesticides, Fellbach, Germany (2015)

EC, 2019a. Commission implementing regulation (EU) 2019/533 concerning a coordinated multiannual control programme of the Union for 2020, 2021 and 2022 to ensure compliance with maximum residue levels of pesticides and to assess the consumer exposure to pesticide residues in and on food of plant and animal origin. Off. J. Eur. Union L88/28.

EC, 2019b. SANTE/12682/2019. Guidance Document on Analytical Quality Control and Method Validation Procedures for Pesticide Residues and Analysis in Food and Feed. 2019b. European Commission. Directorate General for Health and Food Safety.

Elliott, J.E., Kirk, D.A., Martin, P.A., Wilson, L.K., Kardosi, G., Lee, S., McDaniel, T., Hughes, K.D., Smith, B.D., Idrissi, A.M., 2018. Effects of halogenated contaminants on reproductive development in wild mink (Neovison vison) from locations in Canada. Ecotoxicology 27, 539-555.

EN. European Norm 15662, 2019. Foods of Plant Origin - Multimethod for the Determination of Pesticide Residues Using GC- and LC-based Analysis Following Acetonitrile Extraction/Partitioning and Clean-up by Dispersive SPE-modular QuEChERS-method. European Commitee for Standardization.

Encarnacao, T., Pais, A.A., Campos, M.G., Burrows, H.D., 2019. Endocrine disrupting chemicals: impact on human health, wildlife and the environment. Sci. Prog. 102, $3-42$.

Espin, S., Garcia-Fernandez, A.J., Herzke, D., Shore, R.F., van Hattum, B., Martinez-Lopez, E., Coeurdassier, M., Eulaers, I., Fritsch, C., Gomez-Ramirez, P., Jaspers, V.L., Krone, O., Duke, G., Helander, B., Mateo, R., Movalli, P., Sonne, C., van den Brink, N.W., 2016. Tracking pan-continental trends in environmental contamination using sentinel raptors-what types of samples should we use? Ecotoxicology 25, 777-801.

Espin, S., Terraube, J., Arroyo, B., Camarero, P.R., Mateo, R., Liminana, R., VazquezPumarino, X., Pinilla, A., Garcia, J.T., Mougeot, F., 2018. Blood concentrations of p,p'DDE and PCBs in harriers breeding in Spain and Kazakhstan. Sci. Total Environ. 624, 1287-1297. 
Fajardo, I., Ruiz, A., Zorrilla, I., Valero, A., Fernández, I., Sáez, E., Molino, F.M., Olivares, J. 2012. Use os specialised canine units to detect poisoned baits and recover forensic evidence in Andalucía (Southern Spain). In: Richards, N. (Ed.), Carbofuran and Wildlife Poisoning: Global Perspectives and Forensic Approaches. John Wiley \& Sons, Inc.

Fox, G.A., 2001. Wildlife as sentinels of human health effects in the Great Lakes-St. Lawrence basin. Environ. Health Perspect. 109 (Suppl. 6), 853-861.

Garcia-Heras, M.S., Arroyo, B., Simmons, R.E., Camarero, P.R., Mateo, R., Mougeot, F., 2018. Blood concentrations of PCBs and DDTs in an avian predator endemic to southern Africa: associations with habitat, electrical transformers and diet. Environ. Pollut. 232, 440-449.

Haines, D.A., Saravanabhavan, G., Werry, K., Khoury, C., 2017. An overview of human biomonitoring of environmental chemicals in the Canadian Health Measures Survey: 2007-2019. Int. J. Hyg. Environ. Health 220, 13-28.

Henriquez-Hernandez, L.A., Carreton, E., Camacho, M., Montoya-Alonso, J.A., Boada, L.D., Bernal Martin, V., Falcon Cordon, Y., Falcon Cordon, S., Zumbado, M., Luzardo, O.P. 2017. Potential role of pet cats as a sentinel species for human exposure to flame retardants. Front. Vet. Sci. 4, 79.

Hernandez, M., Margalida, A., 2008. Pesticide abuse in Europe: effects on the Cinereous vulture (Aegypius monachus) population in Spain. Ecotoxicology 17, 264-272.

Hernout, B.V., Arnold, K.E., McClean, C.J., Grimm, V., Boxall, A.B., 2011. Predicting the threats of chemicals to wildlife: what are the challenges? Integr. Environ. Assess. Manag. 7, 499-501.

Ibarluzea, J., Aurrekoetxea, J.J., Porta, M., Sunyer, J., Ballester, F., 2016. The biomonitoring of toxic substances in biological samples of general population. Gac. Sanit. 30 (Suppl. 1), 45-54.

Jaspers, V.L., Sonne, C., Soler-Rodriguez, F., Boertmann, D., Dietz, R., Eens, M., Rasmussen, L.M., Covaci, A., 2013. Persistent organic pollutants and methoxylated polybrominated diphenyl ethers in different tissues of white-tailed eagles (Haliaeetus albicilla) from West Greenland. Environ. Pollut. 175, 137-146.

Ji, C., Song, Q., Chen, Y., Zhou, Z., Wang, P., Liu, J., Sun, Z., Zhao, M., 2020. The potential endocrine disruption of pesticide transformation products (TPs): the blind spot of pesticide risk assessment. Environ. Int. 137, 105490.

Klich, D., Lopucki, R., Stachniuk, A., Sporek, M., Fornal, E., Wojciechowska, M., Olech, W., 2020. Pesticides and conservation of large ungulates: health risk to European bison from plant protection products as a result of crop depredation. PLoS One 15, e0228243.

Krief, S., Berny, P., Gumisiriza, F., Gross, R., Demeneix, B., Fini, J.B., Chapman, C.A., Chapman, L.J., Seguya, A., Wasswa, J., 2017. Agricultural expansion as risk to endangered wildlife: pesticide exposure in wild chimpanzees and baboons displaying facial dysplasia. Sci. Total Environ. 598, 647-656.

Lehotay, S.J., Son, K.A., Kwon, H., Koesukwiwat, U., Fu, W., Mastovska, K., Hoh, E., Leepipatpiboon, N., 2010. Comparison of QuEChERS sample preparation methods for the analysis of pesticide residues in fruits and vegetables. J. Chromatogr. A 1217, $2548-2560$.

Liao, J.Y., Fan, C., Huang, Y.Z., Pei, K.J., 2019. Distribution of residual agricultural pesticides and their impact assessment on the survival of an endangered species. J. Hazard. Mater. 121871.

Luzardo, O.P., Ruiz-Suarez, N., Almeida-Gonzalez, M., Henriquez-Hernandez, L.A., Zumbado, M., Boada, L.D., 2013. Multi-residue method for the determination of 57 persistent organic pollutants in human milk and colostrum using a QuEChERSbased extraction procedure. Anal. Bioanal. Chem. 405, 9523-9536.

Luzardo, O.P., Boada, L.D., Carranza, C., Ruiz-Suarez, N., Henriquez-Hernandez, L.A., Valeron, P.F., Zumbado, M., Camacho, M., Pérez-Arellano, J.L., 2014a. Socioeconomic development as a determinant of the levels of organochlorine pesticides and PCBs in the inhabitants of Western and Central African countries. Sci. Total Environ. 497-498, 97-105.

Luzardo, O.P., Ruiz-Suárez, N., Henríquez-Hernández, L.A., Valerón, P.F., Camacho, M. Zumbado, M., Boada, L.D., 2014b. Assessment of the exposure to organochlorine pesticides, PCBs and PAHs in six species of predatory birds of the Canary Islands, Spain. Sci. Total Environ. 472, 146-153.

Luzardo, O.P., Ruiz-Suarez, N., Valeron, P.F., Camacho, M., Zumbado, M., HenriquezHernandez, L.A., Boada, L.D., 2014c. Methodology for the identification of 117 pesticides commonly involved in the poisoning of wildlife using GC-MS-MS and LC-MSMS. J. Anal. Toxicol. 38 (3), 155-163.

Luzardo, O.P., Almeida-Gonzalez, M., Ruiz-Suarez, N., Zumbado, M., HenriquezHernandez, L.A., Meilan, M.J., Camacho, M., Boada, L.D., 2015. Validated analytical methodology for the simultaneous determination of a wide range of pesticides in human blood using GC-MS/MS and LC-ESI/MS/MS and its application in two poisoning cases. Sci. Justice 55, 307-315.

Malarvannan, G., Poma, G., Covaci, A., 2020. Interspecies comparison of the residue levels and profiles of persistent organic pollutants in terrestrial top predators. Environ. Res. 183, 109187.

Malinowska, E., Jankowski, K., 2015. Pesticide residues in some herbs growing in agricultural areas in Poland. Environ. Monit. Assess. 187, 775

Moreno-Gonzalez, R., Rodriguez-Mozaz, S., Huerta, B., Barcelo, D., Leon, V.M., 2016. Do pharmaceuticals bioaccumulate in marine molluscs and fish from a coastal lagoon? Environ. Res. 146, 282-298.

Motas-Guzman, M., Marla-Mojica, P., Romero, D., Martinez-Lopez, E., Garcia-Fernandez, A.J., 2003. Intentional poisoning of animals in southeastern Spain: a review of the veterinary toxicology service from Murcia, Spain. Vet. Hum. Toxicol. 45, 47-50.

Mutengwe, M.T., Chidamba, L., Korsten, L., 2016. Pesticide residue monitoring on South African fresh produce exported over a 6-year period. J. Food Prot. 79, 1759-1766.
Nakayama, S.M.M., Morita, A., Ikenaka, Y., Mizukawa, H., Ishizuka, M., 2019. A review: poisoning by anticoagulant rodenticides in non-target animals globally. J. Vet. Med. Sci. 81, 298-313.

Ntemiri, K., Saravia, V., Angelidis, C., Baxevani, K., Probonas, M., Kret, E., Mertzanis, Y. Iliopoulos, Y., Georgiadis, L., Skartsi, D., Vavylis, D., Manolopoulos, A., Michalopoulou, P., Xirouchakis, S.M., 2018. Animal mortality and illegal poison bait use in Greece. Environ. Monit. Assess. 190, 488.

Obimakinde, S., Fatoki, O., Opeolu, B., Olatunji, O., 2017. Veterinary pharmaceuticals in aqueous systems and associated effects: an update. Environ. Sci. Pollut. Res. Int. 24 3274-3297.

Ogada, D.L., 2014. The power of poison: pesticide poisoning of Africa's wildlife. Ann. N. Y. Acad. Sci. 1322 (1) 1-20.

Ortiz-Santaliestra, M.E., Resano-Mayor, J., Hernandez-Matias, A., Rodriguez-Estival, J. Camarero, P.R., Moleon, M., Real, J., Mateo, R., 2015. Pollutant accumulation patterns in nestlings of an avian top predator: biochemical and metabolic effects. Sci. Total Environ. 538, 692-702.

Orton, F., Lutz, I., Kloas, W., Routledge, E.J., 2009. Endocrine disrupting effects of herbicides and pentachlorophenol: in vitro and in vivo evidence. Environ. Sci. Technol. 43 (6), 2144-2150.

Peng, X., Zheng, K., Liu, J., Fan, Y., Tang, C., Xiong, S., 2018. Body size-dependent bioaccumulation, tissue distribution, and trophic and maternal transfer of phenolic endocrine-disrupting contaminants in a freshwater ecosystem. Environ. Toxicol. Chem. 37, 1811-1823.

Plaza, P.I., Martinez-Lopez, E., Lambertucci, S.A., 2019. The perfect threat: pesticides and vultures. Sci. Total Environ. 687, 1207-1218.

Qie, M., Zhao, Y., Yang, S., Wang, W., Xu, Z., 2019. Rapid simultaneous determination of 160 drugs in urine and blood of livestock and poultry by ultra-high-performance liquid chromatography-tandem mass spectrometry. J. Chromatogr. A 1608, 460423.

Reif, J.S., 2011. Animal sentinels for environmental and public health. Public Health Rep. 126 (Suppl. 1), 50-57.

Ruiz-Suarez, N., Henriquez-Hernandez, L.A., Valeron, P.F., Boada, L.D., Zumbado, M. Camacho, M., Almeida-Gonzalez, M., Luzardo, O.P., 2014. Assessment of anticoagulant rodenticide exposure in six raptor species from the Canary Islands (Spain). Sci. Total Environ. 485-486, 371-376.

Ruiz-Suarez, N., Boada, L.D., Henriquez-Hernandez, L.A., Gonzalez-Moreo, F., SuarezPerez, A., Camacho, M., Zumbado, M., Almeida-Gonzalez, M., Del Mar Travieso-Aja, M., Luzardo, O.P., 2015. Continued implication of the banned pesticides carbofuran and aldicarb in the poisoning of domestic and wild animals of the Canary Islands (Spain). Sci. Total Environ. 505, 1093-1099.

Sage, M., Fourel, I., Coeurdassier, M., Barrat, J., Berny, P., Giraudoux, P., 2010. Determination of bromadiolone residues in fox faeces by LC/ESI-MS in relationship with toxicological data and clinical signs after repeated exposure. Environ. Res. 110, 664-674.

Sanchez-Barbudo, I.S., Camarero, P.R., Mateo, R., 2012. Primary and secondary poisoning by anticoagulant rodenticides of non-target animals in Spain. Sci. Total Environ. 420, 280-288

Sathishkumar, P., Meena, R.A.A., Palanisami, T., Ashokkumar, V., Palvannan, T., Gu, F.L., 2020. Occurrence interactive effects and ecological risk of diclofenac in environmental compartments and biota - a review. Sci. Total Environ. 698, 134057.

Scientific Committee On Consumer S, Bernauer, U., 2016. Opinion of the Scientific Committee on Consumer Safety (SCCS) - revision of the opinion on o-phenylphenol, sodium o-phenylphenate and potassium o-phenylphenate (OPP), in cosmetic products. Regul. Toxicol. Pharmacol. 79, 105

Seljetun, K.O., Eliassen, E., Madslien, K., Viljugrein, H., Vindenes, V., Oiestad, E.L., Moe, L. 2019. Prevalence of anticoagulant rodenticides in feces of wild red foxes (Vulpes vulpes) in Norway. J. Wildl. Dis. 55, 834-843.

Sell, B., Sniegocki, T., Zmudzki, J., Posyniak, A., 2018. Development of an analytical procedure for the determination of multiclass compounds for forensic veterinary toxicology. J. Anal. Toxicol. 42, 183-191.

Shin, Y., Lee, J., Lee, J., Lee, J., Kim, E., Liu, K.H., Lee, H.S., Kim, J.H., 2018. Validation of a multiresidue analysis method for 379 pesticides in human serum using liquid chromatography-tandem mass spectrometry. J. Agric. Food Chem. 66, 3550-3560.

Srivastava, A., Rai, S., Kumar Sonker, A., Karsauliya, K., Pandey, C.P., Singh, S.P., 2017. Simultaneous determination of multiclass pesticide residues in human plasma using a mini QuEChERS method. Anal. Bioanal. Chem. 409, 3757-3765.

SWGTOX, 2013. Scientific Working Group for Forensic Toxicology (SWGTOX) standard practices for method validation in forensic toxicology. J. Anal. Toxicol. 37, 452-474.

Taliansky-Chamudis, A., Gomez-Ramirez, P., Leon-Ortega, M., Garcia-Fernandez, A.J. 2017. Validation of a QuECheRS method for analysis of neonicotinoids in small volumes of blood and assessment of exposure in Eurasian eagle owl (Bubo bubo) nestlings. Sci. Total Environ. 595, 93-100.

Vijayasarathy, S., Baduel, C., Hof, C., Bell, I., Del Mar Gomez Ramos, M., Ramos, M.J.G., Kock M., Gaus, C., 2019. Multi-residue screening of non-polar hazardous chemicals in green turtle blood from different foraging regions of the Great Barrier Reef. Sci. Total Environ. 652, 862-868.

Wang, Y., Guo, B., Gao, Y., Xu, P., Zhang, Y., Li, J., Wang, H., 2014. Stereoselective degradation and toxic effects of benalaxyl on blood and liver of the Chinese lizard Eremias argus. Pestic. Biochem. Physiol. 108, 34-41.

WWF, 2018. In: Grooten, M., Almond, R.E.A. (Eds.), Living Planet Report-2018: Aiming Higher. World Wildlife Fund, Gland, Switzerland (2018). 